\title{
Evaluating Focused Ion Beam and Ultramicrotome Sample Preparation for Analytical Microscopies of the Cathode Layer of a Polymer Electrolyte Membrane Fuel Cell
}

\author{
Lis G. de A. Melo, ${ }^{1}$ Adam P. Hitchcock, ${ }^{2,3\left({ }^{*}\right)}$ Viatcheslav Berejnov, ${ }^{4}$ Darija Susac, ${ }^{4}$ Juergen \\ Stumper $^{4}$ and Gianluigi A. Botton ${ }^{1,2 *}$ \\ 1. Dept. of Materials Science \& Engineering, McMaster University, Hamilton, ON L8S 4M1 Canada \\ 2. Brockhouse Institute for Materials Research, McMaster University, Hamilton, ON L8S 4M1 Canada \\ 3. Dept of Chemistry \& Chemical Biology, McMaster University, Hamilton, ON L8S 4M1 Canada \\ 4. AFCC - Automotive Fuel Cell Cooperation Corp. Burnaby, BC Canada V5J 5J8
}

(*) corresponding author. Tel. 1-905-525-9140, ext 24749. Fax. 1-905-521-2773. Email. aph@ mcmaster.ca

\begin{abstract}
Optimizing the structure of the porous electrodes of polymer electrolyte membrane fuel cells (PEM-FC) can improve device power and durability. Analytical microscopy techniques are important tools for measuring the electrode structure, thereby providing guidance for structural optimization. Transmission Electron Microscopy (TEM), with either Energy Dispersive X-Ray (EDX) or Electron Energy Loss Spectroscopy (EELS) analysis, and Scanning Transmission X-Ray Microscopy (STXM) are complementary methods which, together, provide a powerful approach for PEM-FC electrode analysis. Both TEM and STXM require thin (50-200 nm) samples, which can be prepared either by Focused Ion Beam (FIB) milling or by embedding and ultramicrotomy. Here we compare TEM and STXM spectromicroscopy analysis of FIB and ultramicrotomy sample preparations of the same PEM-FC sample, with focus on how sample preparation affects the derived chemical composition and spatial distributions of carbon support and ionomer. The FIB lamella method, while avoiding pore-filling by embedding media, had significant problems. In particular, in the FIB sample the carbon support was extensively
\end{abstract}


amorphized and the ionomer component suffered mass loss and structural damage. Although each sample preparation technique has a role to play in PEM-FC optimization studies, it is important to be aware of the limitations of each method.

Keywords: PEM-FC; ionomer; electron microscopy; scanning transmission X-ray microscopy; focused ion beam; microtomy

J. Power Sources (submitted 22 Oct 2015; revised manuscript submitted 29 Jan 2016)

File: CCM-FIB-damage.doc 29 Jan 2016 


\section{Introduction}

The rate limiting reaction in a Polymer Electrolyte Membrane Fuel Cell (PEM-FC) is the oxygen reduction reaction (ORR), which occurs in the cathode catalyst layer at the complex triple-phase boundary between two electron conductive phases (carbon support and Pt/Pt alloy nanoparticles), a proton conductor phase (perfluorosulfonic acid (PFSA) ionomer), and a gas phase porous structure penetrating through the carbon support. The effectiveness of the electrochemical reaction that converts chemical into electrical energy depends on the structural relationship among these materials and how they affect transport of protons, oxidant and product water. Optimizing the cathode structure to perform an electrochemical reaction with minimum transport resistances leads to maximization of the fuel cell performance and durability.

One of the main challenges is to understand what influences the transport of the reactants to the catalyst reaction sites and the removal of product water from the pores. Effective modeling of the transport properties relies on accurate information on the microstructure of the catalyst layer [1]. Visualization of the distribution of each component in the cathode catalyst layer can be achieved with various electron and x-ray microscopies. The main challenge has been to characterize the nanoscale distribution of the ionomer in the active layer since the ionomer is highly susceptible to radiation damage [2-4]. The PFSA ionomer influences the electrochemical active surface area, mass transport, both the proton and electron conductance, the porosity of the electrode layer, and acts as a binding agent in the catalyst layer. The PEM-FC membrane, separating the cathode and anode, is chemically similar to the ionomer. Both components are copolymers made up of a hydrophobic tetrafluoroethylene backbone with good mechanical properties, and hydrophilic ionic side chains, responsible for proton conduction. 
Optimizing the ionomer spatial distribution in the catalyst layer is critical to improving the performance of the fuel cell. This also helps minimize costs by maximizing the fraction of the Pt catalyst that can be accessed by protons and $\mathrm{O}_{2}$, thereby allowing design of catalyst layers with low Pt loading. The main PFSA ionomer used in PEM-FC is Nafion ${ }^{\circledR}$, which is available with varying size of side chains, molecular weights and chain terminations. Paul et al. [5] suggested that there may be a difference between proton conductivity of bulk PFSA membrane and the PFSA ionomer present in the catalyst layer due to the reorganization of the nanostructure of PFSA when it is in very thin (sub-10 nm) films. It is unknown exactly how the nanoscale morphology of the PFSA is related to proton and $\mathrm{O}_{2}$ transport [6].

Transmission Electron Microscopy (TEM) in both bright field and atomic-weightsensitive, High Angular Annular Dark Field (HAADF) modes, as well as Electron Energy Loss Spectroscopy (EELS) and Energy Dispersive X-ray Spectroscopy (EDX) have been applied to image the PFSA membrane and ionomer at sub-5 nm scale [2,4,6-9]. In order to mitigate the effects of radiation damage, the nanostructure of PFSA membranes has been imaged with electron microscopy using cryo-techniques [2,9]. Since carbon-containing materials have similar contrast in HAADF images, the ionomer in PEM-FC electrodes has been imaged using stained samples $[4,8]$ where ionomer contrast in enhanced.

Soft X-ray spectromicroscopy has also been applied to map distributions of ionomer relative to the catalyst and support structure in the cathode $[10,11]$. Scanning Transmission XRay Microscopy (STXM) is a synchrotron-based technique that has successfully visualized the ionomer in catalyst coated membranes (CCMs). This was achieved by spectroscopically mapping and differentiating the ionomer, carbon support and embedding epoxy, through $\mathrm{C} 1 \mathrm{~s}$ and $\mathrm{F} 1 \mathrm{~s}$ near edge X-ray absorption (NEXAFS) signals [10-14]. STXM is an ideal tool to study ionomer 
in CCM, since the radiation damage caused by soft X-rays per unit of analytical information is much lower than the analogous electron microscopy technique, TEM-EELS with core loss spectroscopy [15-17]. TEM-EELS and STXM both cause radiation damage and result in similar chemical changes for similar deposited doses [14-16]. However, in X-ray absorption each absorbed X-ray provides analytically useful spectral information, whereas deposition of the equivalent energy through inelastic electron scattering results in a very large number of valence ionization events, which break bonds but do not provide the analytically useful core loss information.

A serious limitation to analytical microscopy methods is sample preparation artefacts. A variety of methods $[18,19]$ have been used to prepare the thin samples $(50-200 \mathrm{~nm})$ needed for both transmission electron and x-ray microscopy techniques. Ultramicrotomy has been used successfully to prepare uniformly thin sections of PEM-FC CCMs [18]. However, loosely connected parts of the sample (for example carbon black particles) can be detached and smeared over the section during slicing [20]. Also, structural alterations can occur due to mechanical stresses [21] deforming the morphology and phase transformation properties of the ionomer [6]. Microtoming CCM samples which combine hard (carbon support/Pt particles) and soft (ionomer) materials may result in cutting artefacts [22]. These issues can affect the correct assessment of the microstructure and the interpretation of the results. Another disadvantage is the need to embed the material. Finding a suitable embedding material and preparation strategy is challenging [18,19]. Partial penetration of the cathode by the embedding material is ideal [19], but the degree of penetration depends on the porosity and the surface tension of the material. Embedding and ultramicrotomy are not always reproducible and can lead to distortions of the nanostructure due to swelling of the polymerizing embedding material in the cathode layer pores. 
Another technique frequently used to prepare thin specimens is Focused Ion Beam (FIB) milling [23]. 3D pore size distributions and connectivity of the carbon support, both pristine [24] and after electrochemical testing [25], have been determined from FIB in combination with Scanning Electron Microscopy (SEM) in slice-and-view serial FIB tomography. This is valuable information that can improve modeling of the mass transport in the electrodes [1]. However, there are concerns that the nanostructure may be modified by FIB milling. During FIB milling, the material is ablated by a gallium ion beam, which removes material and modifies the surface of the remaining sample. This may result in local amorphization in insulating or semi-conducting materials; defects or intermetallic phases for certain metals $[23,26]$; heating damage; Ga implantation [26]; sample re-deposition; and the curtain effect in polymer composites [27]. For soft materials, FIB can cause serious chemical and physical transformation of the sample, such as knock-on damage and radiolysis [21,22]. Structural changes such as chain scission, cross-linking, chain shrinkage can transform the structure and change the crystallinity of polymers.

Nonetheless FIB sample preparation has been applied successfully to some soft samples [28], including bacterial samples with attached biominerals [29], a hard-soft system with some properties analogous to PEM-FC cathodes.

Local heating is a major concern during FIB milling of polymers as it can result in significant chemical change $[21,28,30]$. Heating damage depends on the current and accelerating voltages of the ion and electron beams, and also on the thermal conductivity of the sample, as only a small portion of the incident kinetic energy is responsible for breaking the bonds and milling the sample $[21,27,28]$. This damage is usually minimized by using low beam currents [21,28], cryostages [28], or alternative scanning techniques [21,30]. Structural transformations of FIB milled polymeric samples (for example polyacrylamide and lignite coal) have been analyzed 
using STXM at the C 1s edge [21]. In that work, it was reported that the SEM electron beam used for imaging was a larger cause of damage than the ion beam [21]. For polycarbonate, phase contrast in atomic force microscopy (AFM) images was related to the ion beam energy and elastic modulus of the polymer, allowing a determination that $25 \mathrm{keV}$ was the ion beam energy that would minimize FIB damage [31]. Semiconducting polymers suffer extensive damage during FIB milling, with the main effects being chain scission and cross-linking with disruption of the conjugated system [32].

Different polymers exhibit different changes when damaged in FIB milling, reflecting differences in their chemistry. Because of the increased use of FIB for sample preparation and 3D imaging of PEM-FC using slice-and-view, it is important to understand and quantify the damage done by FIB to the different chemical components, in comparison to the alternative of embedding and ultramicrotome sample preparation. Although there are some studies of FIB damage to soft materials [21,31], the rate and types of damage for FIB milling of PEM-FC materials has not been characterized to our knowledge. Several groups, including the present authors, have identified FIB damage as a concern [20,24,33], but we are unaware of any quantitative in-depth study. The objective of this work is to compare microstructural properties of all components in the cathode catalyst layer, especially the ionomer, for samples of the same CCM prepared with ultramicrotomy and FIB. Morphological details were analyzed with TEM bright field and HAADF imaging; elemental analysis was performed with EDX and EELS measured in scanning transmission electron microscopy (STEM) mode; detailed spectromicroscopy studies were carried out using near edge X-ray absorption spectral contrast (NEXAFS) in STXM. EELS and EDX in TEM and the $\mathrm{C}$ 1s and F 1s NEXAFS in STXM showed there was extensive damage to the carbon support in the FIB milled CCM sample. 
Damage to the ionomer in the FIB milled sample was extremely severe: mass loss and structural damage were observed and quantified with STXM. The advantage of preparing the CCM with FIB is the possibility of preparing thinner and more uniform sections than with ultramicrotomy. However, these results indicate that FIB samples of PEM-FC CCMs are likely to be missing the key component - ionomer. This paper is organized as follows: Section 2 describes the experimental protocols used; Section 3 presents the results, first from TEM methods, and then from STXM; Section 4 presents the discussion, followed by a short summary.

\section{Experimental procedures}

\subsection{Sample Preparation}

The sample used is a CCM with an 18 micron thick Gore-SELECTA® ePTFE microreinforced membrane. The cathode had a $0.4 \mathrm{mg} / \mathrm{cm}^{2} \mathrm{Pt}$ loading on a graphitized carbon catalyst support. The Pt deposition process used a hexachloro Pt acid precursor. Two sample preparation techniques were applied to the same sample: ultramicrotoming and Focused Ion Beam (FIB) milling. In order to reduce penetration of the embedding material into the cathode in the ultramicrotomy sample, high MW polystyrene (PS) was used [10]. The CCM was sandwiched between two PS beads which had been gently softened by a brief exposure to toluene vapor. The composite was dried overnight at $60^{\circ} \mathrm{C}$. The microtomed slices were cut at room temperature with a Leica Ultracut UCT at a thickness of approximately $100 \mathrm{~nm}$ with a DiATOME diamond knife. Sections were transferred from a water surface to holey carbon coated 200 mesh Cu grids (Electron Microscopy Sciences). A cryo-microtomy section was also prepared by soaking the sample overnight in a sucrose solution [34] then flash freezing to $-130^{\circ} \mathrm{C}$, followed by cryomicrotoming. The cryo-cut section was transferred to a grid, which was subsequently floated in a 
water bath to dissolve the sucrose.

For the FIB preparation, a cross-section of the same CCM was glued with carbon tape and silver paste onto a $90^{\circ} \mathrm{Al} \mathrm{SEM} \mathrm{stub.} \mathrm{The} \mathrm{region} \mathrm{of} \mathrm{interest} \mathrm{was} \mathrm{the} \mathrm{cathode} \mathrm{material.} \mathrm{A} \mathrm{W}$ strip was applied to the top surface of the sample over the area of interest to minimize artifacts and control rate of removal. More details on FIB sample preparation can be found in the literature [35]. A Zeiss NVision40 FIB was operated with a $\mathrm{Ga}^{+}$beam at $30 \mathrm{kV}$ and $80 \mathrm{pA}$ ion beam current. The sample was at room temperature. Lower ion beam energies are known to reduce the thickness of amorphous layers in FIB milled Si [23]. A lower polishing ion beam voltage and current were used $(10 \mathrm{kV}, 40 \mathrm{pA})$ with the intention of reducing sample damage.

\subsection{TEM, EDX, Tomography and TEM-EELS}

All TEM imaging and analytical measurements were carried out using a field emission JEOL 2010F TEM/STEM operated at $200 \mathrm{keV}$. The EDX detector (Oxford Instruments, Inca model 6498 with area detector $30 \mathrm{~mm}^{2}$ and ATW window) had a collection solid angle of $0.1 \mathrm{Sr}$. A Gatan imaging filter (GIF-Tridiem Ultrascan 2Kx2K) was used for EELS. For electron tomography, a specialized Fischione holder (model 2020) was used on a FEI Company Titan 80300 TEM, a high resolution aberration corrected HRTEM/STEM operated at $300 \mathrm{keV}$ in scanning mode. The FEI software Xplore3D was used for STEM tomography calibration and acquisition. The tilt series was taken over $0^{\circ}$ to $-64^{\circ}$ and then from $1^{\circ}$ to $+65^{\circ}$ with $2^{\circ}$ increment. The convergence angle was $5.5 \mathrm{mrad}$. An electron beam current of $55 \mathrm{pA}$ was used. The camera length used for acquiring both tilt series was $115 \mathrm{~mm}$ leading to an inner scattering angle collected on the Fischione High-Angle Annular Dark-Field (HAADF) detector of $50 \mathrm{mrad}$. Automated Fourier filter cross-correlation alignment and manual alignment of the tilt axis were 
used to align the images of the tilt series using the software Inspect $3 \mathrm{D}^{\mathrm{TM}}$. Both weighted back projection (WBP) reconstruction and simultaneous iterative reconstruction technique (SIRT, 25 iterations) algorithms were used. The Amira ${ }^{\mathrm{TM}}$ software was used to visualize the reconstructed volume.

\subsection{STXM}

The FIB and ultramicrotomed specimens were measured using STXMs on beamline 10ID1 at the Canadian Light Source (CLS) [36] and beamline 5.3.2.2 at the Advanced Light Source (ALS) [37]. The STXM instrumentation, acquisition and analysis have been presented elsewhere $[38,39]$. The $\mathrm{C}$ 1s and $\mathrm{F}$ 1s edges were of particular interest since these are the majority elements of the ionomer. Among the chemical species in a CCM, the F 1s signal is unique to the PFSA ionomer and membrane components. The C 1s NEXAFS spectra readily distinguish the ionomer from the carbon support, the polystyrene (PS) and the holey carbon support [10]. Image sequences (also called stacks [40]), were acquired at the C 1s and F 1s edges for the cathode region of the microtome and FIB sections.

The transmitted signal measured in STXM is converted to optical density, OD $=-\ln \left(\mathrm{I} / \mathrm{I}_{\mathrm{o}}\right)$, where $\mathrm{I}$ is the signal transmitted through the sample and $\mathrm{I}_{\mathrm{o}}$ is the incident photon intensity, measured from the transmitted signal without the sample, but with all other components in the path (e.g. a holey carbon or formvar substrate, or a hole for the FIB free standing sample). The OD can then be quantitatively related to type, density and thickness of the sample, through $\mathrm{OD}(\mathrm{E})=\Sigma \mu_{\mathrm{i}}(\mathrm{E}) \rho_{\mathrm{i}} \cdot \mathrm{t}_{\mathrm{i}}$ where $\mu_{\mathrm{i}}(\mathrm{E})$ is the mass-absorption coefficient and $\rho_{\mathrm{i}}$ is the density of the $\mathrm{i}^{\text {th }}$ material component and $t_{i}$ is the thickness of that component at a specific point in the sample. If the chemical composition and density of each material are known and a spectrum of the pure material is available, the absolute thickness of each component at every position in a sample can 
be determined by fitting the spectrum at each position to a weighted sum of a suitable set of reference spectra, each of which are scaled to an absolute intensity scale expressed as optical density per $1 \mathrm{~nm}$ thickness (OD1) [39]. The spectral weight of each component at each pixel are assembled into quantitative component maps [39]. This approach was used for the microtomed sample where there was negligible sample damage. However there were significant chemical and possibly density changes in the FIB preparation (as characterized by this work), which meant that we could not apply this method to the FIB sample. Instead, the carbon and fluorine components were quantified by matching reference spectra extracted from the sample to the $\mathrm{C} 1 \mathrm{~s}$ and F 1s signals tabulated for the elements [41], and an assumed density of $1.0 \mathrm{~g} . \mathrm{cm}^{-3}$. This assumption converts the derived material thicknesses into effective rather than absolute values, still allowing comparative evaluation of the relative amounts of ionomer and carbon support in the microtomed and FIB samples. This does not affect the results of our damage analysis as the spectroscopy clearly reveals dramatic changes of the material as well as overall OD signal changes.

All STXM data analysis was performed using the aXis2000 software [42]. As with other additive analytical methods, one must account for each component present. In practice, that is done by carefully evaluating the suitability of internal or external reference spectra and checking that the statistical quality of the singular value decomposition (SVD), which is an efficient matrix method equivalent to an optimized linear least squares fit for highly overdetermined data sets [43], is within that expected from the overall statistical quality of the data [39].

Internal reference spectra were generated by either finding isolated regions of the pure component of a given sample or by extracting the pure spectrum from the spectrum of a region with two components which can be differentiated and thus deconvolved using component- 
specific spectral features (e.g. the $\mathrm{C} 1 \mathrm{~s} \rightarrow \pi^{*}$ peak at $285.2 \mathrm{eV}$ of the carbon support). For each analysis, internal reference spectra were generated and compared with other spectra previously acquired $[10,12,14]$. Since the internal reference spectra of the carbon support region always contains contributions from the ionomer, Pt catalyst and PS embedding material, it was necessary to subtract a weighted amount of the spectral signature of the ionomer, PS and Pt in order to isolate the spectrum of the pure carbon support material in the microtome section. Prior knowledge of the $\mathrm{C} 1 \mathrm{~s}$ spectrum expected for graphitic carbon support, as well as guidance from the fit to the elemental response for carbon, were used to guide the amounts of PFSA, PS and constant signals that were subtracted to generate the internal, pure carbon support spectrum for the microtome section. After isolating internal reference spectra of each component, they were converted to a quantitative intensity scale (optical density per $1 \mathrm{~nm}, \mathrm{OD} 1$ ) by scaling the intensity of the isolated internal reference spectrum so that, outside of the near-edge spectral structure energy range, it matched the intensity of the X-ray absorption predicted from the elemental composition, standard density and elemental absorption coefficients of the material [41]. The OD1 reference spectra were then used to fit the spectrum at each pixel of the C-1s edge stack in order to generate quantitative components maps.

For analysis of the $\mathrm{C} 1 \mathrm{~s}$ image sequence of the FIB sample, it was possible to extract the spectrum of a damaged carbon support component from a small area, while the majority of the $\mathrm{C}$ 1s signal was that of amorphous carbon. The spectrum of the amorphous carbon component was converted to OD1 by scaling the intensity considering only the carbon elemental response with an assigned density of $1 \mathrm{~g} . \mathrm{cm}^{-3}$. This density was arbitrarily chosen as we do not know the actual composition or density of the FIB damaged carbon component. The residual of the analysis indicated a successful fit, and the absence of a pattern indicated that the carbon in the FIB section 
was mostly amorphous carbon, with very little crystalline carbon support.

The quantification of the fluorine content in both FIB and microtomed samples was done by first selecting internal reference spectra for PFSA from the membrane of the microtome sample. Although the C 1s and F 1s spectra of membrane and cathode ionomer may differ, our NEXAFS studies have shown that the differences are small, much smaller than the changes in the $\mathrm{C}$ 1s and F 1s spectra caused by FIB damage. Since the membrane is present in all our samples, and is chemically similar to the ionomer, we took the spectrum of the membrane as representative of the ionomer. This spectrum was set to a quantitative OD1 intensity scale by scaling to match the elemental response for PFSA. For the FIB section, the F 1s signal from the entire region of the FIB section was used and a quantitative OD1 intensity was set by scaling its intensity to that for elemental fluorine with an assigned density of $1 \mathrm{~g} . \mathrm{cm}^{-3}$. By using the same (arbitrary) density for the $\mathrm{C}$ and $\mathrm{F}$ elemental response the relative amounts will be correct, even though the derived absolute thicknesses will be skewed from the actual amount by the density of the amorphous ion beam damaged material. A pixel-by-pixel fit to the $\mathrm{F} 1 \mathrm{~s}$ image sequence yielded quantitative component maps for the ionomer (microtome sample) or residual fluorinated material (FIB section), and the non-fluorinated components (as the constant in an image sequence analysis).

\section{Results \& Discussion}

\subsection{TEM characterization of FIB versus microtomed samples}

\subsubsection{Amorphization of carbon support}

The cathode catalyst layer consists of Pt nanoparticles on $\sim 50 \mathrm{~nm}$ graphitic carbon support particles, with an ionomer layer between the support particles, ideally of appropriate 
thickness and in intimate contact with the Pt catalyst. TEM bright field-images of the microtomed section (Figure 1a) clearly show highly ordered graphitic layers in the carbon support particles. In contrast, high resolution imaging of the FIB sample (Figure 1b, 1c) shows no evidence of graphitic order. Rather, the carbon support is extensively disordered and in an amorphous carbon state (see below for spectroscopic support). Amorphization is a common damage effect of FIB in other materials $[23,44]$. We speculate that the damage to the carbon particles in a CCM cathode may be particularly severe due to the porous nature of the material, facilitating the penetration of the ions into the internal surfaces of the section.

These results show that FIB causes severe damage to the carbon support at the nanoscale. It is not clear if there is also a change in porosity, which involves material re-structuring, typically on the micron scale. Schulenburg et al. (2011) [25] determined approximately the same porosity for CCM samples milled at room temperature compared to that in a CCM-sample milled under cryo-conditions using $30 \mathrm{kV}$ FIB-SEM serial tomography. They believed that the high electron and heat conductivity of the catalyst layer prevented significant degradation of the porous structure of the catalyst structure during FIB milling. However, PFSA membranes [45] and single layer PEMFC [46] have low thermal conductivities. Additionally, it has been shown that cryo-FIB of polymers with low thermal conductivity delays, but does not prevent, local heating while milling [30]. Therefore, it is possible that the porosity of the catalyst layer changed during FIB milling, which would affect measurements made with FIB-serial tomography, but this is not within the scope of this paper.

\subsubsection{Gallium contamination}


HAADF images of the FIB section (Figure 2a), which provide contrast based on atomic number, show the presence of additional smaller particles approximately $2 \mathrm{~nm}$ in size or smaller. These particles are implanted Ga, as confirmed by EDX (see maps in Figure 2b). High resolution TEM images, obtained at $80 \mathrm{kV}$ to ensure minimal knock-on electron beam damage, show that there is an $\sim 5 \mathrm{~nm}$ thick amorphous layer at the edge of the carbon support particles which is embedded with Ga clusters $(\sim 1 \mathrm{~nm})$ seen as small dark spots, and Pt nanoparticles ( 5nm) (Fig. 1c). This amorphous layer is a consequence of ion implantation, which results from knock-on damage events in which $\mathrm{Ga}^{+}$ions displace atoms in the target material [47].

Electron tomography was used to determine the 3-D characteristics of the sample and to assess the extent of Ga implantation by overcoming the projection problem of transmission 2-D images (Figure 2c and 2d). Figure 2d is an orthoslice image of the reconstructed volume from the centre of the FIB section. It shows that the Pt particles sit on the surface of the hollow carbon support, consistent with earlier work [48]. Although the carbon support is not visible, the carbon particles are defined by bright edges in the orthoslice, which are Ga particles sitting on the surface of the carbon support. The Ga particles thus help visualize the location of the carbon support particles. The ionomer component is not visible; any ionomer that survived the FIB milling (see below) was probably destroyed during the long acquisition of the tomography tilt series. Tilt series were also acquired for the microtome section. However the tomography data set for the microtomed section could not be analyzed because the sample deformed so extensively during the acquisition that tilt series alignment could not be performed successfully.

EELS spectrum imaging, linescan and point spectra were acquired for both FIB and microtomed sections. EELS spectrum imaging results for the FIB section are shown in Figure 3. Maps of C, F, O and Pt are presented in Figure 3b. The Pt map was estimated from the 
spectrum images in the $\mathrm{C} 1 \mathrm{~s}$ pre-edge region $(215-270 \mathrm{eV})$, where heavy metals dominate the signal due to their large electron density and thus strong valence ionization signal. This approach is similar to a method used in XAS to estimate the distribution of Pt in fuel cell MEAs [12].

EELS spectra for the $\mathrm{C} 1 \mathrm{~s}, \mathrm{O} 1 \mathrm{~s}$ and $\mathrm{F} 1 \mathrm{~s}$ edges, averaged over the spectrum image, are shown in Figure 3c. The $\mathrm{C}$ 1s spectrum clearly indicates the carbon is extensively amorphized, as also found by STXM-XAS. The F 1s signal was very weak but detectable, indicating that a F/C (and thus ionomer/carbon support) ratio and ratio map can be derived. However, the spectral shape is broad and different from that known to be characteristic of PFSA as studied by XAS [10-12]. Although the EELS spectra were acquired with lower energy resolution (roughly $0.5 \mathrm{eV}$ ) than STXM $(0.15 \mathrm{eV})$, the results are consistent with those from STXM-XAS of the same FIB sample (see Figure 7). The C 1s map indicates homogenous distributions. From the F 1s map, it seems that the ionomer distribution is homogenous at the spatial scale shown. This suggests it may be possible to use TEM-EELS to map the ionomer in the active layer at a nanometer scale, but only within the radiation damage limitations [2,49], and only for uniform sections, less than $50 \mathrm{~nm}$ thick. On the other hand, fluorine was not detected in the cathode of the microtomed sample using EELS. This could have happened because we were not sufficiently careful to limit the electron beam dose to a level which would avoid radiation damage and mass loss of the ionomer component. Since similar acquisition strategies were used for the TEM-EELS mapping of the FIB sample which did see a clear F 1s signal, it is possible that the chemically transformed ionomer component that survived FIB was much harder to break down further.

\subsection{STXM analysis}

The optimum optical density (OD) for STXM using transmission detection is $\sim 1$ which 
corresponds to a thickness of $\sim 100 \mathrm{~nm}$ for C $1 \mathrm{~s}$ spectra of solids with a density of $\sim 1 \mathrm{~g} . \mathrm{cm}^{-3}$. Therefore, reliable quantitative analysis can be achieved with STXM for both FIB and microtome sections. C 1s and F 1s stacks of the microtome and FIB samples were acquired with STXM. Since the different carbon-containing components have different electronic structure and thus different $\mathrm{C}$ 1s spectra, it is possible to map each chemical component based on their characteristic NEXAFS spectrum. The image stacks were fit on a pixel-by-pixel basis with suitable reference spectra using Singular Value Decomposition [43]. However, for damaged samples such as the FIB section this approach has additional uncertainties since the chemical composition and structure of the damaged materials are not known.

Figure 4 presents the analysis of a $\mathrm{C} 1 \mathrm{~s}$ image sequence recorded for the microtome section. The $\mathrm{C} 1 \mathrm{~s}$ reference spectra for each component, derived from the stack as outlined in the experimental section, are displayed in Figure $4 \mathbf{a}$ on an absolute intensity scale (OD per nm of material). All of the carbon containing components - PS, ionomer, carbon support, holey carbon are distinguished and mapped. The analysis fit the spectrum at each pixel to the set of OD1 reference spectra (Fig. 4a) plus a constant, where the constant fits those species without a C 1s signal (corresponding to the Pt catalyst for the microtome section - see [12]). The quantitative maps for each chemical component generated by the fit are shown in Figure $\mathbf{4 b}$, with grey scales on an absolute nanometre thickness scale. The nm thickness scale for the Pt component was generated from the constant map and the pre-C1s OD1 response using the method described elsewhere [39]. The PFSA component map is dominated by the strong signal from the membrane (located in the top right corner of the region sampled). The PFSA signal from the membrane was subtracted and the remaining ionomer signal displayed on an amplified thickness scale, showing in considerable detail the distribution of the ionomer in the cathode. 
Figure 5 presents the results from analysis of a C 1s image sequence for the FIB sample. The reference spectra were extracted from the stack. One isolated region with a strong $\mathrm{C} 1 \mathrm{~s} \rightarrow \pi^{*}$ transition and some evidence of the $\mathrm{C} 1 \mathrm{~s} \rightarrow \sigma^{*}$ transition was found and the intensity of the pre and post edges from the spectrum of that region were matched to that of graphitic carbon support (as in Fig. 4a). Almost all regions of the FIB sample exhibited a $\mathrm{C} 1 \mathrm{~s}$ spectrum with a relatively weak and broad $\pi^{*}$ signal and a very broad featureless $\sigma^{*}$ region, very typical of amorphous carbon [50]. The intensity scale of the $\mathrm{C}$ 1s spectrum of the FIB sample was converted to a quantitative scale assuming a composition of pure $C$ and a density of $2.2 \mathrm{~g} / \mathrm{cm}^{2}$. Fits were attempted with the inclusion of the reference spectrum of PFSA but there were no regions identifiable as containing unmodified ionomer. In particular, the characteristic $\sigma^{*}(\mathrm{C}-\mathrm{F})$ peaks at $292 \mathrm{eV}$ and $296 \mathrm{eV}$ for PFSA (see Fig. 4a) were not seen anywhere in the FIB section. Thus the component maps reported are those for crystalline carbon support (Fig. 5b), amorphous carbon (Fig. 5c) and the constant (Fig. 5d), which is associated with Pt catalyst, as well as Ga and W from the FIB sample preparation. Figure 5e is a color composite of the carbon support, amorphous carbon and constant component maps. It indicates that the FIB section consists mostly of amorphous carbon. The STXM results show unambiguously that the FIB procedure is amorphizing the sample, with very little evidence of the characteristic spectra of the original components, which are seen clearly in the microtomed sample (Fig. 4).

Analysis of the F 1s STXM results, which are most specific to the ionomer component, are presented in Figure 6 for the microtomed sample and Figure 7 for the FIB section. For the cathode of the microtomed sample, only one shape of F 1s spectrum was observed (Fig. 6a), and the F 1s spectrum in the cathode region, that of the ionomer, was indistinguishable from the spectrum of the membrane, and of PFSA membranes studied previously $[10,12]$. The F 1 s stack 
was processed using stack fit analysis (SVD) fit with a constant component) with the PFSA reference spectrum being that of the membrane, converted to optical density per nm thickness (OD1) using a chemical formula of $\mathrm{C}_{21} \mathrm{~F}_{41} \mathrm{O}_{5} \mathrm{SH}$ and a density of $2.1 \mathrm{~g} . \mathrm{cm}^{-3}$. The output of the fit consisted of a PFSA component map and a map of the constant signal (Fig. 6d), which represents all the non-fluorinated components in the system. The PFSA component map was split into the membrane (Fig. 6b) and ionomer (Fig. 6c) components using an intensity threshold technique which readily identifies the cathode-membrane boundary. The color-coded composite map (Fig. 6e) correlates the positions of the ionomer with that of the non-fluorinated components. The results of the stack fit analysis of the F 1s stack of the FIB section are shown in Figure 7. The two F 1s spectra used in the fit are presented in Figure 7a. One spectrum is the average over the entire area of the F $1 \mathrm{~s}$ image sequence while the other is the F $1 \mathrm{~s}$ spectrum of PFSA from the microtomed section. Each spectrum was background subtracted and scaled such that the pre-edge and far continuum intensities match those in the predicted X-ray absorption spectrum for elemental fluorine with a density of $1 \mathrm{~g} / \mathrm{cm}^{2}$. Only F was included, and a unit density was used since neither the composition nor the actual density of the highly ion-beam damaged material are known. The component maps of damaged ionomer (average $\mathrm{F} 1 \mathrm{~s}$ spectrum) (Fig. 7b), possibly undamaged ionomer (component fit by the spectrum of PFSA (Fig. 7c), and the constant (non-F components) (Fig. 7d) are shown. The color composite map (Fig. 7e) indicates that there are isolated region of metals (probably Pt) shown in red, and a few hot spots with higher content of less damaged ionomer (in blue), while most of the material is damaged ionomer (green). The integrated signal of less-damaged ionomer (fit by the PFSA F 1s spectrum) is less than $10 \%$ of that of the damaged ionomer (fit by the average F $1 \mathrm{~s}$ spectrum of the FIB section). The spectrum extracted from the pixels reporting less damaged ionomer, which 
is presented in the supplemental material as Figure S1, is still very much modified from that of undamaged PFSA, but not as distorted as the average spectrum.

The average $\mathrm{C} 1 \mathrm{~s}$ and $\mathrm{F} 1 \mathrm{~s}$ spectra of the FIB and microtomed samples are compared in

Figure 8. The areas from which the averaged spectra were acquired are shown as dotted rectangles superimposed on images at $300 \mathrm{eV}$ in Figures 8a and $\mathbf{8 b}$ for the FIB and microtomed samples, respectively. Figure 8c compares the average C 1s spectra while Figure 8d compares the average $\mathrm{F} 1 \mathrm{~s}$ spectra. A PS contribution of $35 \mathrm{~nm}$ was subtracted from the $\mathrm{C} 1 \mathrm{~s}$ microtomed section spectrum in order to meaningfully compare the spectrum with that of the FIB section. The additional offset in the FIB spectra relative to the corresponding microtomed spectra is probably due to absorption by $\mathrm{Ga}$ and $\mathrm{W}$ in the FIB section. These heavy metals also absorb Xrays but do not generate $\mathrm{C} 1 \mathrm{~s}$ or $\mathrm{F} 1 \mathrm{~s}$ signals. The difference in the fine structure of the $\mathrm{C}$ 1s edge for both sections is due to both amorphization and loss of PFSA ionomer in the FIB preparation.

In order to compare the F 1s spectral shape (Figure 8d), the underlying background was subtracted from each spectrum and the data replotted to full scale (Fig. 8e). The C 1s and F 1s spectra of the PFSA ionomer are similar to that of poly(tetrafluoroethylene) (PTFE) [51,52], as expected since PFSA consists of a PTFE backbone. The shape and relative intensity of the 689 and $694 \mathrm{eV}$ peaks are different in the $\mathrm{F} 1 \mathrm{~s}$ spectrum of the FIB section as compared to the $\mathrm{F} 1 \mathrm{~s}$ spectrum of the microtome section, indicating a different C-F bonding environment. The $689 \mathrm{eV}$ peak is much broader, particularly on the lower energy side, indicating a range of chemical environments. The observation of F 1s signal indicates there is still some fluorine in the FIB sample but the change in spectral shape indicates those $\mathrm{F}$ atoms are in a different local environment than the $\mathrm{F}$ atoms in the ionomer in the microtome sample. 
In order to more accurately characterize the extent of loss of fluorine caused by FIB milling, an elemental analysis was performed by combining the $\mathrm{C} 1 \mathrm{~s}$ and $\mathrm{F} 1 \mathrm{~s}$ signals from the same area. The procedure and results are presented in the Supplemental Material. This analysis showed the volumetric F/C ratio for the FIB section was $\sim 0.06 \mathrm{vol} / \mathrm{vol}$ while that for the microtomed section was $\sim 0.3 \mathrm{vol} / \mathrm{vol}$. The significantly lower effective volumetric $\mathrm{F} / \mathrm{C}$ ratio for the FIB sample indicates extensive mass loss of PFSA ionomer during FIB milling (see Figure S2). The effective carbon and ionomer thicknesses in the microtomed and FIB samples (on a nominal $\mathrm{nm}$ scale) are presented in Table 1. The relatively high amount of carbon support in the cathode of the microtomed sample is attributed to interference from the PS embedding component. This is not surprising since the characteristic C $1 \mathrm{~s} \rightarrow \pi^{*}$ peaks for PS and carbon support overlap. When a non-aromatic epoxy is used where there is no such peak overlap, the carbon and fluorine effective thicknesses are similar, resulting in F/C 1.0 (results not shown). The presence of PS in the electrode does not affect the F (ionomer) measurements, since PS does not contain fluorine. Table 1 shows that the total thickness of the FIB and microtomed samples are similar (106 nm and $100 \mathrm{~nm}$, respectively) whereas the total amount of fluorine in the FIB sample is 4 - 5 times less, and loses the characteristic ionomer spectroscopic fingerprint at the same time.

It is possible that the particular location where the FIB section was prepared could have had an anomalously low amount of ionomer, since there is evidence from other work of localized depletion of ionomer in some CCMs [12]. In order to test this hypothesis, another FIB section was prepared and analysed. The second FIB section was prepared using lower $\mathrm{Ga}^{+}$current in the final polishing steps in an attempt to reduce ion beam damage. However in the second FIB section there was absolutely no F 1s signal detected in the F 1s STXM image sequence. In 
addition, different microtomed sections of the same sample (microtome 1 and 2), and different regions in the same section (areas a and b), as well as a cryo-cut of the same sample were analyzed to characterize the variation in ionomer content. The results are shown in Table $\mathbf{1}$. The amounts of ionomer and carbon support in the microtome sections was determined using two complementary methods: (i) from the average over all cathode pixels in both $\mathrm{C} 1 \mathrm{~s}$ and $\mathrm{F}$ 1s stack maps [10-12]. The C 1s stack map consisted of the difference of OD images at 283.6 and $278 \mathrm{eV}$, and was corrected for contributions of the PS and holey carbon. The F 1s stack map consisted of the difference of OD images at 694 and $684 \mathrm{eV}$. (ii) from ionomer and carbon support component maps extracted by stack fits from C $1 \mathrm{~s}$ and $\mathrm{F} 1 \mathrm{~s}$ image sequences. In the microtomed sections the amount of ionomer relative to the amount of carbon support in the cathode varied between $0.20-0.44 \mathrm{vol} / \mathrm{vol}$. We did not find any regions with only $0.06 \mathrm{vol} / \mathrm{vol}$ ionomer, or with distorted C 1s and F 1s spectra like those observed for the FIB section.

\section{Discussion}

We have compared the structure of the carbon support and the PFSA ionomer in a cathode catalyst layer of the same CCM sample prepared by FIB and ultramicrotome. Although STXM has limited spatial resolution $(\sim 30 \mathrm{~nm})$ compared to TEM, it was of great value in assessing the chemical changes caused by FIB milling. Both TEM/STEM and STXM analysis show that the FIB milling causes a) extensive amorphization of the carbon support; ) structural damage; and c) loss of the PFSA ionomer. In addition to not being damaged by the sample preparation, ultramicrotome samples are easier and less expensive to produce than FIB samples. However, even with the PS sandwich preparation method, there is significant infiltration of embedding material into the cathode of the microtomed samples. The challenge of achieving 
uniform sections with the much smaller thicknesses that are needed for EELS is that CCM samples combine a very soft, rubbery membrane with a much harder carbon support/Pt catalyst layer so that, in the resulting section, either the membrane is very thick, or it is not present at all.

FIB milling damage in fuel cell samples has been assessed previously but only with SEM secondary electron images. The membrane material deteriorated with the FIB milling during a FIB-SEM tomography study [24]. Less surface damage occurred when FIB milling was performed with the CCM at $\sim 100 \mathrm{~K}$, as compared to room temperature FIB [20]. However Raman spectroscopy showed that performing FIB of conjugated polymers under cryogenic conditions does not prevent degradation of $\mathrm{C}=\mathrm{C}$ bonds [32].

There are only a few detailed spectroscopic studies of damage associated with FIB milling of soft materials [21,32]. The chemical changes associated with FIB milling of PFSA are quite different from those observed with conjugated organic polymers and other radiation resistant polymers $[21,32]$. Shift of the $\pi^{*}(C=C)$ and $\sigma^{*}(C-C)$ peaks towards higher energy, attributed to disruption of the conjugated system, was observed with TEM-EELS of a FIB milled poly-3-hexathiophene thin conjugated polymer film [32]. For lignite coal and polyacrylamide, spectral changes indicating formation of new bonds were attributed to SEM imaging taken during FIB milling rather than the ion beam [21]. The FIB-induced damage observed in this work was a lot larger than the damage observed for FIB preparation of samples of less radiation sensitive polymers $[21,32]$.

FIB milling of soft materials can result in damage by beam-induced heating and knock-on displacement [21,28,32]. Beam-induced heating occurs over a few tens of nanometers [21] and is negligible for most hard materials [47]. The amount of localized heating also depends on beam current, ion voltage and the thermal conductivity of the sample [21]. Due to the low thermal 
conductivity of the PFSA ionomer [46], it is possible that the presence of a thin layer of ionomer in the catalyst layer insulates and thus prevents the Pt/carbon support from dissipating the heat of the ion beam, which could then lead to a large increase of temperature that damages the ionomer layer in the cathode. This effect can be even larger when thin lamella samples are made due to the loss of mass of surrounding material that would otherwise contribute to heat dissipation. This would explain the ionomer mass loss shown in Figure 8, S1 and Table 1. The presence of Pt/C particles has been reported to affect electron beam radiation damage on fuel cell samples with varying amounts of PFSA [53].

Thermal analysis studies have been reported for bulk PFSA in the acid form [54] and in different gaseous environments [55]. Basically, PFSA membranes are thermally stable to approximately $300^{\circ} \mathrm{C}$, where the sulfonic acid groups are lost. Eventually PTFE backbone decomposition with high mass loss occurs around $500^{\circ} \mathrm{C}$ [54-56]. Determination of the exact temperature rise during FIB milling is not trivial and depends on several factors, such as thermal conductivity of the sample, beam power, geometry of the sample and thermal sinking [57]. Temperatures increase up to $1000^{\circ} \mathrm{C}$ have been measured using a nanothermo-probe on a thermocouple junction undergoing FIB milling [58]. Localized temperature rises of over $2000^{\circ} \mathrm{C}$ have been calculated for soft materials (e.g. PMMA), whereas the corresponding calculated temperature rise for silicon is only $100^{\circ} \mathrm{C}$, due to its much higher thermal conductivity [59].

Chemical alteration from knock-on damage by energetic Ga ions is usually constrained to a thin surface layer of FIB-prepared sample, where the Ga displaces the atoms from the sample [48]; a 'pristine' core is normally expected to exist in the centre of the sample [21]. However, in porous materials, such as the catalyst layer in a PEMFC, it is possible that the Ga ions penetrate much deeper than in a nonporous sample, resulting in FIB causing extensive damage throughout 
the lamella thickness. This effect may preclude accepted methods for minimizing FIB damage, such as using lower energy/current in the final polishing steps or using different ion beam scanning techniques, both of which were useful in reducing damage in FIB of bulk polymer samples [21,28,30,31]. Knock-on damage can destroy functional groups [21], rearrange bonds, and lead to bond-breaking $[60,61]$, all of which can be deduced from the changes in both the C 1s and F 1s spectra of the FIB section (Fig. 4 and 5). The damage to the carbon support is mostly knock-on damage, which causes the amorphization [47] observed with both HR-TEM images (Fig. 1) and in the STXM C 1s spectrum (Fig. 5).

Due to rapid radiation damage by the electron beam, reliable quantification of the ionomer fraction is challenging when using TEM-EDX particularly with low solid angle detectors, which are most commonly used today. State-of-the-art large area detectors can improve detection of ionomer if mass loss radiation damage is minimized using cryo-conditions [2]. TEM-EELS can be used to measure the ionomer distribution in the cathode, as shown in Figure 3 and [2], but there are severe challenges in the preparation of sufficiently thin samples $(<50 \mathrm{~nm})$ so that near single scattering spectra can be acquired, as well as making the EELS measurements with a sufficiently low dose so as to minimize radiation damage. As part of future work, low dose TEM-EELS acquisition strategies will be explored with the goal of acquiring F 1s EELS of a CCM prepared by ultramicrotomy. Quantitative dose-damage relationships in STXM and TEM will be measured so that appropriate, low-damage strategies can be devised, thereby allowing each technique to achieve the best results possible.

It is interesting to reflect on the success of the tomography for the FIB sample, and the lack of success for the microtomed sample. In the latter case, deformation or displacement of the carbon support particles probably occurred due to progressive loss of ionomer due to electron 
beam damage. The successful tomography of the FIB sample suggests that removal of the ionomer in the FIB milling makes the remaining electrode structure stiffer and thus less responsive to further damage by TEM in dose-intensive tomography. Thus the damage caused by FIB milling could be considered an advantage for electron beam tomography studies of porosity in CCM samples, although the pore volume may be overestimated due to changes to the ionomer component.

\section{Conclusions}

In the quest to image ionomer in catalyst layers, proper sample preparation is crucial to achieving valid results as well as nanoscale resolution. Both microtomy and FIB sample preparation techniques impose challenges to this quest. For microtomy there are concerns about possible modifications to the morphology of the sample, smearing of materials during sectioning, possible interference with embedding media, and challenges to achieve a uniform and sufficiently thin section. On the other hand, FIB is excellent at making very thin sections and has been widely used in combination with SEM for serial tomography. However, here we have shown that FIB milling causes morphological damage to the carbon support material as well as extensive differential elemental loss and chemical damage to the ionomer in the cathode, when compared to the microtomed section of the same sample.

Spectromicroscopy techniques are the best available tools to map the nanoscale distributions of ionomer in PEMFC cathodes. This study, using a variety of electron beam and X-ray spectromicroscopies, has shown chemical changes to the PFSA ionomer are induced when using FIB milling relative to ultramicrotome sample preparation. In particular, using highresolution electron microscopy, electron tomography, and analytical methods in TEM and 
STXM we have demonstrated the detailed evolution of the structure of the carbon support and the PFSA ionomer in the cathode of a CCM sample. The amorphization of the carbon support and presence of $\mathrm{Ga}$ at its surface was shown with bright field TEM, HAADF images and electron tomography, and quantified with C 1s NEXAFS in STXM. The changes in the chemical environment through mass loss and changes in the bonding of both carbon and fluorine atoms in the original PFSA ionomer after FIB milling were revealed by comparing $\mathrm{C} 1 \mathrm{~s}$ and $\mathrm{F} 1 \mathrm{~s}$ spectra of the FIB sample to those of the microtomed sample. The issues raised in this paper should be taken into account when quantifying and analyzing the chemical composition of cathode catalyst layers of PEM-FC.

\section{Acknowledgements}

This research was supported by NSERC (Discovery Grants to APH and GAB and the Automotive Partnership Program CaRPE-FC network), CFI, and the Canada Research Chair program. We thank Marcia Reid (Health Sciences Microscopy) and Julia Huang (CCEM) for expert sample preparation. We thank Andreas Korinek and Carmen Andrei for help with TEM acquisition at the CCEM. The Canadian Centre for Electron Microscopy (CCEM) is a Canadian national facility supported by NSERC, the Canada Foundation for Innovation under the MSI program and McMaster University. Research described in this paper was performed in part at the Canadian Light Source, which is supported by the Canadian Foundation for Innovation, Natural Sciences and Engineering Research Council of Canada, the University of Saskatchewan, the Government of Saskatchewan, Western Economic Diversification Canada, the National 
Research Council Canada, and the Canadian Institutes of Health Research. Results were also acquired at beamline 5.3.2.2 at the ALS, which is supported by the Director of the Office of Science, Department of Energy, under Contract No. DE-AC02-05CH11231. 


\section{References}

[1] S. Kim, H. Pitsch, Reconstruction and effective transport properties of the catalyst layer in PEM fuel cells, J. Electrochem. Soc. 156 (2009) B673. doi:10.1149/1.3106136.

[2] D.A. Cullen, R. Koestner, R.S. Kukreja, Z.Y. Liu, S. Minko, O. Trotsenko, et al., Imaging and Microanalysis of Thin Ionomer Layers by Scanning Transmission Electron Microscopy, J. Electrochem. Soc. 161 (2014) F1111-F1117. doi:10.1149/2.1091410jes.

[3] F. Scheiba, N. Benker, U. Kunz, C. Roth, H. Fuess, Electron microscopy techniques for the analysis of the polymer electrolyte distribution in proton exchange membrane fuel cells, J. Power Sources. 177 (2008) 273-280. doi:10.1016/j.jpowsour.2007.11.085.

[4] M. Lopez-Haro, L. Guétaz, T. Printemps, A. Morin, S. Escribano, P.-H. Jouneau, et al., Three-dimensional analysis of Nafion layers in fuel cell electrodes, Nat. Commun. 5 (2014) 5229. doi:10.1038/ncomms6229.

[5] D. Paul, A. Fraser, K. Karan, Towards the understanding of proton conduction mechanism in PEMFC catalyst layer: Conductivity of adsorbed Nafion films, Electrochem. Commun. 13 (2011) 774-777. doi:10.1016/j.elecom.2011.04.022.

[6] S. Yakovlev, K.H. Downing, Visualization of Clusters in Polymer Electrolyte Membranes by Electron Microscopy, Phys. Chem. Chem. Phys. 15 (2012) 1052-64. doi:10.1039/c2cp42969a.

[7] S. Yakovlev, X. Wang, P. Ercius, N.P. Balsara1, K.H. Downing, Direct Imaging of Nanoscale Acidic Clusters in a Polymer Electrolyte Membrane, J Am Chem Soc. 133 (2011) 20700-20703. doi:10.1021/ja209240d.Direct.

[8] L. Guetaz, S. Escribano, O. Sicardy, Study by electron microscopy of proton exchange membrane fuel cell membrane-electrode assembly degradation mechanisms: Influence of local conditions, J. Power Sources. 212 (2012) 169-178. doi:10.1016/j.jpowsour.2012.03.096.

[9] C. Wang, V. Krishnan, D. Wu, R. Bledsoe, S.J. Paddison, G. Duscher, Evaluation of the microstructure of dry and hydrated perfluorosulfonic acid ionomers: microscopy and simulations - SUP, J. Mater. Chem. A. 1 (2013) 938. http://xlink.rsc.org/?DOI=c2ta01034h.

[10] D. Susac, V. Berejnov, A.P. Hitchcock, J. Stumper, STXM Study of the Ionomer Distribution in the PEM Fuel Cell Catalyst Layers, in: ECS Trans., 2011: pp. 629-635. doi:10.1149/1.3635596.

[11] V. Berejnov, D. Susac, J. Stumper, A.P. Hitchcock, 3D Chemical Mapping of PEM Fuel Cell Cathodes by Scanning Transmission Soft X-ray SpectroTomography, ECS Trans. 50 (2013) 361-368. doi:10.1149/05002.0361ecst.

[12] A.P. Hitchcock, V. Berejnov, V. Lee, M. West, V. Colbow, M. Dutta, et al., Carbon corrosion of proton exchange membrane fuel cell catalyst layers studied by scanning transmission X-ray microscopy, J. Power Sources. 266 (2014) 66-78.

doi:10.1016/j.jpowsour.2014.04.119.

[13] V. Lee, D. Susac, S. Kundu, V. Berejnov, R.T. Atanasoski, A.P. Hitchcock, et al., STXM Characterization of Nanostructured Thin Film Anode Before and After Start-Up Shutdown and Reversal Tests, ECS Trans. 58 (2013) 473-479. doi:10.1149/05801.0473ecst.

[14] D. Susac, V. Berejnov, J. Stumper, A.P. Hitchcock, STXM Characterization of PEM Fuel Cell Catalyst Layers, ECS Trans. 50 (2012) 405-413. doi:10.1149/05002.0405ecst.

[15] J. Wang, G.A. Botton, M.M. West, A.P. Hitchcock, Quantitative evaluation of radiation 
damage to polyethylene terephthalate by soft X-rays and high-energy electrons., J. Phys. Chem. B. 113 (2009) 1869-76. doi:10.1021/jp808289e.

[16] E.G. Rightor, A.P. Hitchcock, H. Ade, R.D. Leapman, S.G. Urquhart, A.P. Smith, et al., Spectromicroscopy of Poly(ethylene terephthalate): Comparison of Spectra and Radiation Damage Rates in X-ray Absorption and Electron Energy Loss, J. Phys. Chem. B. 101 (1997) 1950-1960. doi:10.1021/jp9622748.

[17] A.P. Hitchcock, J.J. Dynes, G. Johansson, J. Wang, G. Botton, Comparison of NEXAFS microscopy and TEM-EELS for studies of soft matter, Micron. 39 (2008) 741-748. doi:10.1016/j.micron.2007.09.008.

[18] D.A. Blom, J.R. Dunlap, T.A. Nolan, L.F. Allard, Preparation of Cross-Sectional Samples of Proton Exchange Membrane Fuel Cells by Ultramicrotomy for TEM, J. Electrochem. Soc. 150 (2003) A414. doi:10.1149/1.1556593.

[19] K. More, S. Reeves, TEM Specimen Preparation of Partially-Embedded Electrodes From Proton Exchange Membrane Fuel Cell Membrane Electrode Assemblies, Microsc. Microanal. 11 (2005) 2104-2105. doi:10.1017/S1431927605508377.

[20] C.S. Kuroda, Y. Yamazaki, Novel Technique of MEA Sample Preparation Using a Focused Ion Beam for Scanning Electron Microscope Investigation, in: ECS Trans., ECS, 2007: pp. 509-516. doi:10.1149/1.2780964.

[21] N.D. Bassim, B.T. De Gregorio, A.L.D. Kilcoyne, K. Scott, T. Chou, S. Wirick, et al., Minimizing damage during FIB sample preparation of soft materials, J. Microsc. 245 (2012) 288-301. doi:10.1111/j.1365-2818.2011.03570.x.

[22] G.H. Michler, Special Preparation Techniques, in: Electron Microsc. Polym., Springer Berlin Heidelberg, 2008: pp. 219-230.

[23] J. Mayer, L.A. Giannuzzi, T. Kamino, J. Michael, TEM Sample Preparation and FIB induced Damage, MRS Bull. 32 (2007) 400-407.

[24] C. Ziegler, S. Thiele, R. Zengerle, Direct three-dimensional reconstruction of a nanoporous catalyst layer for a polymer electrolyte fuel cell, J. Power Sources. 196 (2011) 2094-2097. doi:10.1016/j.jpowsour.2010.09.044.

[25] H. Schulenburg, B. Schwanitz, N. Linse, G.G. Scherer, A. Wokaun, J. Krbanjevic, et al., 3D Imaging of Catalyst Support Corrosion in Polymer Electrolyte Fuel Cells, J. Phys. Chem. C. 115 (2011) 14236-14243. doi:10.1021/jp203016u.

[26] C. Lehrer, L. Frey, S. Petersen, M. Mizutani, M. Takai, H. Ryssel, Defects and galliumcontamination during focused ion beam micro machining, in: 2000 Int. Conf. Ion Implant. Technol. Proceedings. Ion Implant. Technol. - 2000 (Cat. No.00EX432), IEEE, 2000: pp. 695-698. doi:10.1109/.2000.924248.

[27] O. Olea-Mejía, O. Olea-Cardoso, R. Lopez-Castañares, FIB-SEM combination technique for characterization of polymer composites, in: A. Méndez-Vilas (Ed.), Curr. Microsc. Contrib. to Adv. Sci. Technol., 2012: pp. 1060-1065. http://www.formatex.info/microscopy5/book/1060-1065.pdf (accessed July 2, 2014).

[28] S. Kim, M. Jeong Park, N.P. Balsara, G. Liu, A.M. Minor, Minimization of focused ion beam damage in nanostructured polymer thin films, Ultramicroscopy. 111 (2011) 191-199. doi:10.1016/j.ultramic.2010.11.027.

[29] M. Obst, P. Gasser, D. Mavrocordatos, M. Dittrich, TEM-specimen preparation of cell/mineral interfaces by Focused Ion Beam milling, Am. Mineral. 90 (2005) 1270-1277. doi:10.2138/am.2005.1743.

[30] R. Schmied, B. Chernev, G. Trimmel, H. Plank, New possibilities for soft matter 
applications: eliminating technically induced thermal stress during FIB processing, RSC Adv. 2 (2012) 6932. doi:10.1039/c2ra21025h.

[31] R.J. Bailey, R. Geurts, D.J. Stokes, F. de Jong, A.H. Barber, Evaluating focused ion beam induced damage in soft materials., Micron. 50 (2013) 51-6.

doi:10.1016/j.micron.2013.04.005.

[32] M. Sezen, H. Plank, E. Fisslthaler, B. Chernev, A. Zankel, E. Tchernychova, et al., An investigation on focused electron/ion beam induced degradation mechanisms of conjugated polymers, Phys. Chem. Chem. Phys. 13 (2011) 20235.

doi:10.1039/c1cp22406a.

[33] L. G. de A. Melo, V. Lee, D. Susac, V. Berejnov, J. Stumper, G.A. Botton, et al., Effects of Sample Preparation Technique on Quantitative Analysis of Automotive Fuel Cell Catalyst Layers, Microsc. Microanal. 20 (2014) 472-473. doi:10.1017/S1431927614004085.

[34] N. Subramaniam, A. Simpson, M.J. Monteiro, O. Shaffer, C.M. Fellows, R.G. Gilbert, Cryo-Sectioning and Chemical-Fixing Ultramicrotomy Techniques for Imaging Rubber Latex Particle Morphology, Microsc. Res. Tech. 63 (2004) 111-114. doi:10.1002/jemt.20018.

[35] R. Anderson, S.J. Klepeis, Practical aspects of FIB tem specimen preparation with emphasis on semiconductor applications, Introd. to Focus. Ion Beams Instrumentation, Theory, Tech. Pract. (2005) 173-200. doi:10.1007/0-387-23313-X_9.

[36] K.V. Kaznatcheev, C. Karunakaran, U.D. Lanke, S.G. Urquhart, M. Obst, a. P. Hitchcock, Soft X-ray spectromicroscopy beamline at the CLS: Commissioning results, Nucl. Instruments Methods Phys. Res. Sect. A Accel. Spectrometers, Detect. Assoc. Equip. 582 (2007) 96-99. doi:10.1016/j.nima.2007.08.083.

[37] A.L.D. Kilcoyne, T. Tyliszczak, W.F. Steele, S. Fakra, P. Hitchcock, K. Franck, et al., Interferometer-controlled scanning transmission X-ray microscopes at the Advanced Light Source, J. Synchrotron Radiat. 10 (2003) 125-136. doi:10.1107/S0909049502017739.

[38] A.P. Hitchcock, H.D.H. Stöver, L.M. Croll, R.F. Childs, Chemical Mapping of Polymer Microstructure Using Soft X-ray Spectromicroscopy, Aust. J. Chem. 58 (2005) 423. doi:10.1071/CH05054.

[39] A.P. Hitchcock, Soft X-Ray Imaging and Spectromicroscopy, in: G. Van Tendeloo, V.D. Dyck, S.J. Pennycook (Eds.), Handb. Nanoscopy Vol.II, Wiley-VCH Verlag GmbH \& Co. KGaA, 2012: pp. 745-791.

[40] C. Jacobsen, S. Wirick, G. Flynn, C. Zimba, Soft x-ray spectroscopy from image sequences with sub-100 nm spatial resolution., J. Microsc. 197 (2000) 173-84. http://www.ncbi.nlm.nih.gov/pubmed/11543408.

[41] B.L. Henke, E.M. Gullikson, J.C. Davis, X-Ray Interactions: Photoabsorption, Scattering, Transmission, and Reflection at $\mathrm{E}=50-30,000 \mathrm{eV}, \mathrm{Z}=1-92$, At. Data Nucl. Data Tables. 54 (1993) 181-342. doi:10.1006/adnd.1993.1013.

[42] A.P. Hitchcock (2015), aXis 2000 is written in Interactive Data Language (IDL). It is available free for non-commercial use from http://unicorn.mcmaster.ca/aXis2000.html.

[43] I.N. Koprinarov, A.P. Hitchcock, C.T. McCrory, R.F. Childs, Quantitative Mapping of Structured Polymeric Systems Using Singular Value Decomposition Analysis of Soft Xray Images, J. Phys. Chem. B. 106 (2002) 5358-5364. doi:10.1021/jp0132811.

[44] L. Giannuzzi, F.A. Stevie, A review of focused ion beam milling techniques for TEM specimen preparation, Micron. 30 (1999) 197-204. doi:10.1016/S0968-4328(99)00005-0. 
[45] P.J.S. Vie, S. Kjelstrup, Thermal conductivities from temperature profiles in the polymer electrolyte fuel cell, Electrochim. Acta. 49 (2004) 1069-1077. doi:10.1016/j.electacta.2003.10.018.

[46] O. Burheim, P.J.S. Vie, J.G. Pharoah, S. Kjelstrup, Ex situ measurements of through-plane thermal conductivities in a polymer electrolyte fuel cell, J. Power Sources. 195 (2010) 249-256. doi:10.1016/j.jpowsour.2009.06.077.

[47] L.A. Giannuzzi, B.I. Prenitzer, B.W. Kempshall, Ion-Solid Interactions, in: L.A. Giannuzzi, F.A. Stevie (Eds.), Introd. to Focus. Ion Beams, Springer US, 2005: pp. 13-52. doi:10.1007/0-387-23313-X_2.

[48] D. Banham, F. Feng, T. Furstenhaupt, K. Pei, S. Ye, V. Birss, Effect of Pt-loaded carbon support nanostructure on oxygen reduction catalysis, J. Power Sources. 196 (2011) 54385445. doi:10.1016/j.jpowsour.2011.02.034.

[49] S. Yakovlev, N.P. Balsara, K.H. Downing, Insights on the study of nafion nanoscale morphology by transmission electron microscopy, Membranes (Basel). 3 (2013) 424-439. doi:10.3390/membranes3040424.

[50] J. Diaz, O. Monteiro, Z. Hussain, Structure of amorphous carbon from near-edge and extended x-ray absorption spectroscopy, Phys. Rev. B. 76 (2007) 1-12. doi:10.1103/PhysRevB.76.094201.

[51] T. Ohta, K. Seki, T. Yokoyama, I. Morisada, K. Edamatsu, Polarized XANES Studies of Oriented Polyethylene and Fluorinated PolyethyIenes, Phys. Scipta. 41 (1990) 150-153.

[52] C. Ziegler, T. Schedel-Niedrig, G. Beamson, D.T. Clark, W.R. Salaneck, H. Sotobayashi, et al., X-Ray Absorption Study of Highly Oriented Poly(tetrafluoroethylene) Thin Films, Langmuir. 10 (1994) 4399-4402. doi:10.1021/la00024a001.

[53] Q. He, J. Chen, D.J. Keffer, D.C. Joy, Electron beam induced radiation damage in the catalyst layer of a proton exchange membrane fuel cell, Scanning. 36 (2013) 338-46. doi:10.1002/sca.21117.

[54] S.H. De Almeida, Y. Kawano, Thermal Behaviour of Nafion Membranes, J. Therm. Anal. Calorim. 58 (1999) 569-577. doi:10.1023/A:1010196226309.

[55] S. Samms, S. Wasmus, R. Savinell, Thermal stability of Nafion ${ }^{\circledR}$ in simulated fuel cell environments, J. Electrochem. Soc. 143 (1996) 1498-1504. http://jes.ecsdl.org/content/143/5/1498.short (accessed July 2, 2014).

[56] J. Surowiec, R. Bogoczek, Studies on the thermal stability of the perfluorinated cationexchange membrane Nafion-417, J. Therm. Anal. 33 (1988) 1097-1102. doi:10.1007/BF01912735.

[57] C.A. Volkert, A.M. Minor, Focused Ion Beam Micromachining, 32 (2007) 389-399.

[58] N. Shukla, S.K. Tripathi, A. Banerjee, A. S.V. Ramana, N.S. Rajput, V.N. Kulkarni, Study of temperature rise during focused $\mathrm{Ga}$ ion beam irradiation using nanothermo-probe, Appl. Surf. Sci. 256 (2009) 475-479. doi:10.1016/j.apsusc.2009.07.024.

[59] R. Schmied, J.E. Fröch, A. Orthacker, J. Hobisch, G. Trimmel, H. Plank, A combined approach to predict spatial temperature evolution and its consequences during FIB processing of soft matter., Phys. Chem. Chem. Phys. 16 (2014) 6153-8. doi:10.1039/c3cp55308f.

[60] A. V Krasheninnikov, F. Banhart, Engineering of nanostructured carbon materials with electron or ion beams., Nat. Mater. 6 (2007) 723-33. doi:10.1038/nmat1996.

[61] F. Banhart, Irradiation effects in carbon nanostructures, Reports Prog. Phys. 62 (1999) 1181-1221. doi:10.1088/0034-4885/62/8/201. 
Table 1 Ionomer to Carbon Support Ratio for FIB and Several Microtomed Samples ${ }^{(a)}$ of the Same CCM Evaluated by STXM

\begin{tabular}{|l|c|c|c|}
\hline (1) Microtomed & \multicolumn{2}{|c|}{ Thickness (nm) } & $\begin{array}{c}\text { Ionomer/C_support } \\
\text { (vol / vol) }\end{array}$ \\
\hline Sample $^{(b)}$ & Ionomer & C_support & $\sim 27 \%$ \\
\hline Cryo-microtome & 12 & 44 & $\sim 44 \%$ \\
\hline Microtome \#1 & 11 & 25 & $\sim 40 \%$ \\
\hline Microtome \#2 area A & 10 & 25 & $\sim 40 \%$ \\
\hline Microtome \#2 area B & 4 & 20 & $\sim 20 \%$ \\
\hline
\end{tabular}

\begin{tabular}{|c|c|c|c|}
\hline (2) FIB, elemental ${ }^{(c)}$ & \multicolumn{2}{|c|}{ Effective thickness (nm) } & \multirow{2}{*}{$\begin{array}{l}\text { Ionomer/C_support } \\
(\mathrm{vol} / \mathrm{vol})\end{array}$} \\
\hline Sample & Ionomer & C_support & \\
\hline Microtome & 25 & 75 & $\sim 33 \%$ \\
\hline FIB & 6 & 100 & $\sim 6 \%$ \\
\hline
\end{tabular}

(a) Microtome \# 1 refers to another microtome section or slice, mounted on a different TEM grid. Area A and Area B refer to different areas within the same microtome section or slices.

(b) Ionomer-to-carbon-support ratio derived from STXM analysis of a cryo-microtome section, two microtome sections and two areas in the same section. The values in the upper table are derived from both C 1s and F 1s stack maps (2 energies [10,14]) and analysis of full C 1s and F 1s stacks.

(c) From fit of the average $\mathrm{C} 1 \mathrm{~s}$ and $\mathrm{F}$ 1s spectra to the elemental reference spectra. The value for

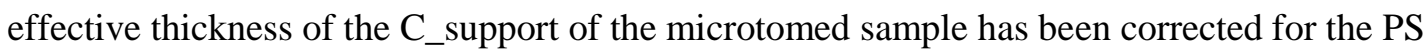
contribution based on the relative amounts of PS and C_support in the cathode determined from the stack fit. 

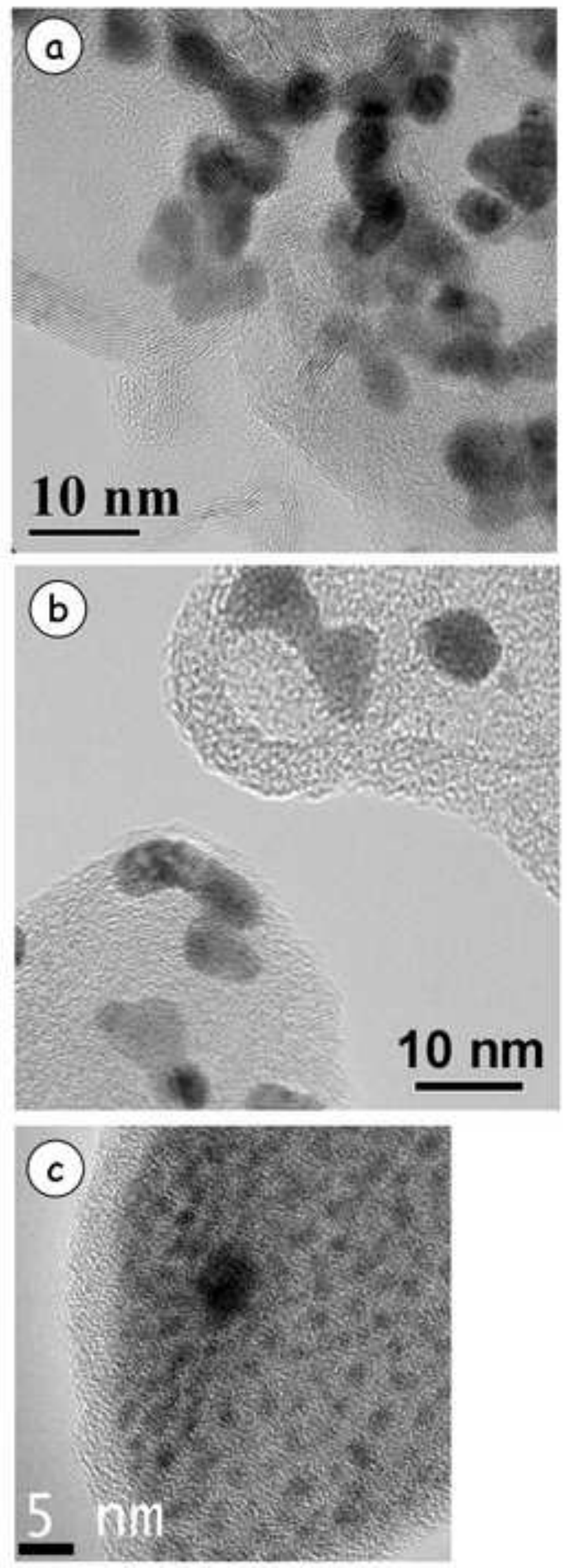

Figure 1

\section{$10 \mathrm{~nm}$}

(c)

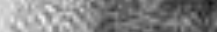

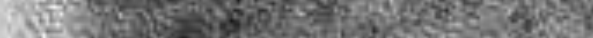

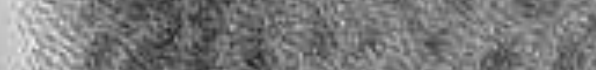

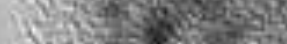

$5 \mathrm{~mm}$ 

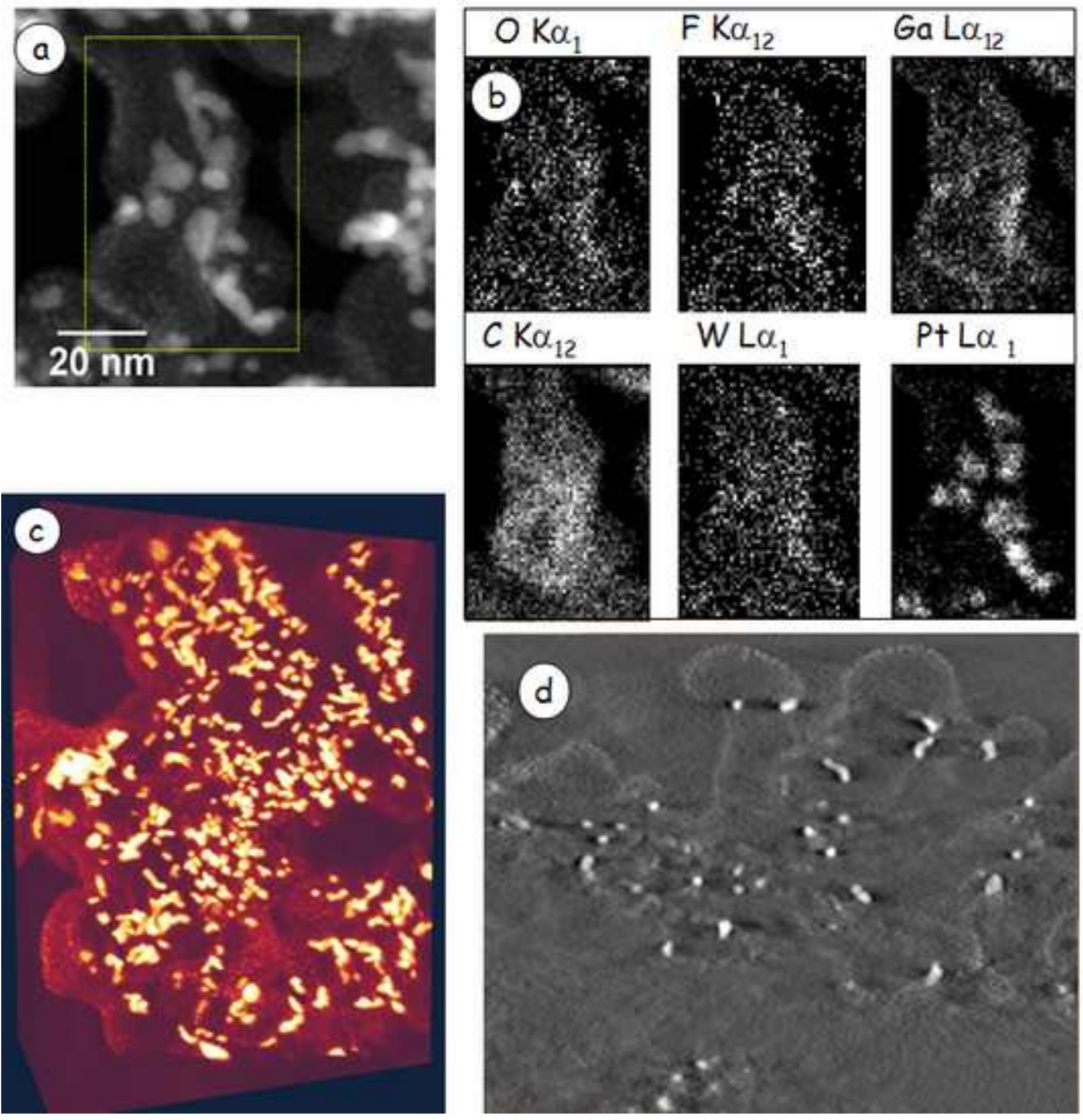

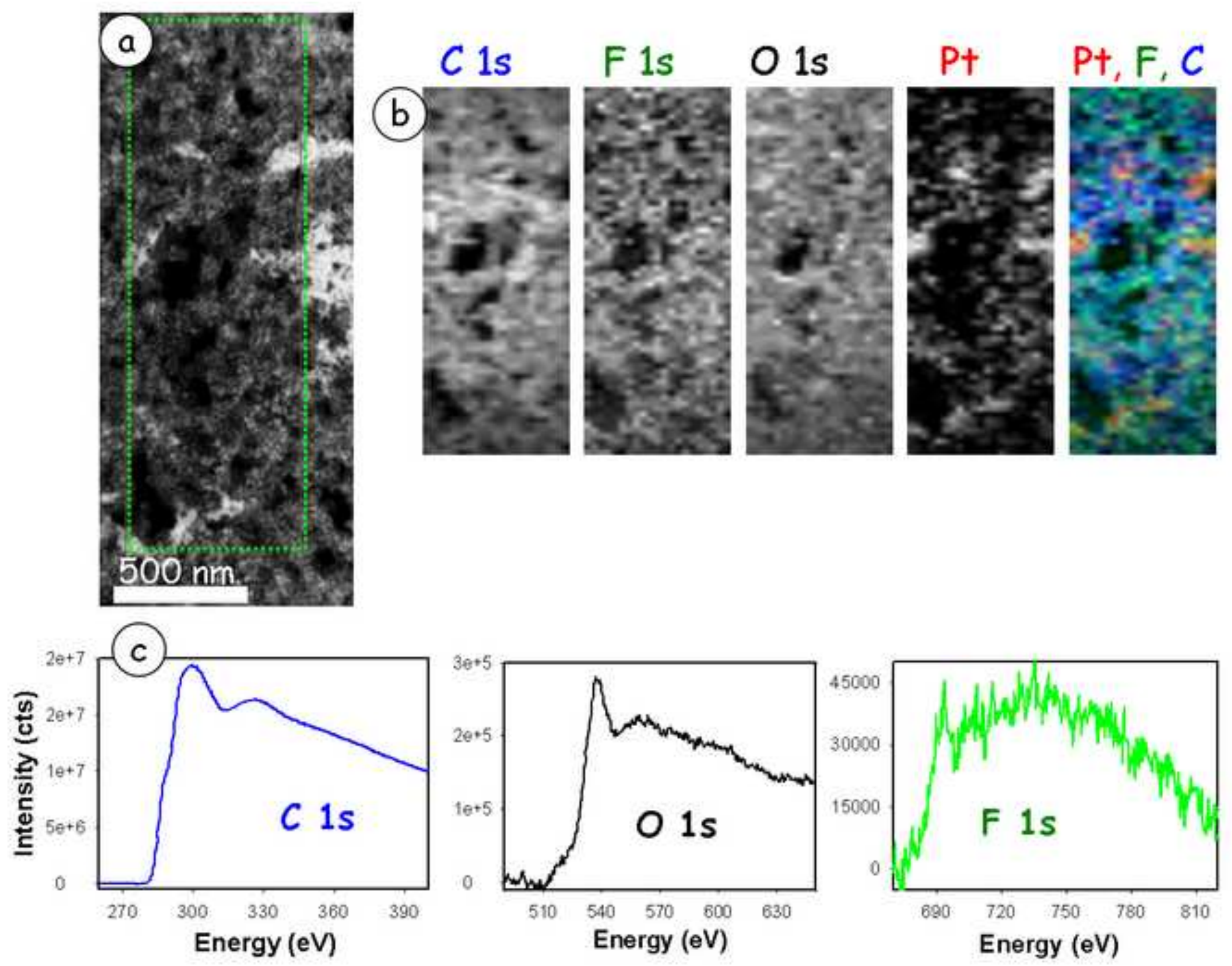
(a)

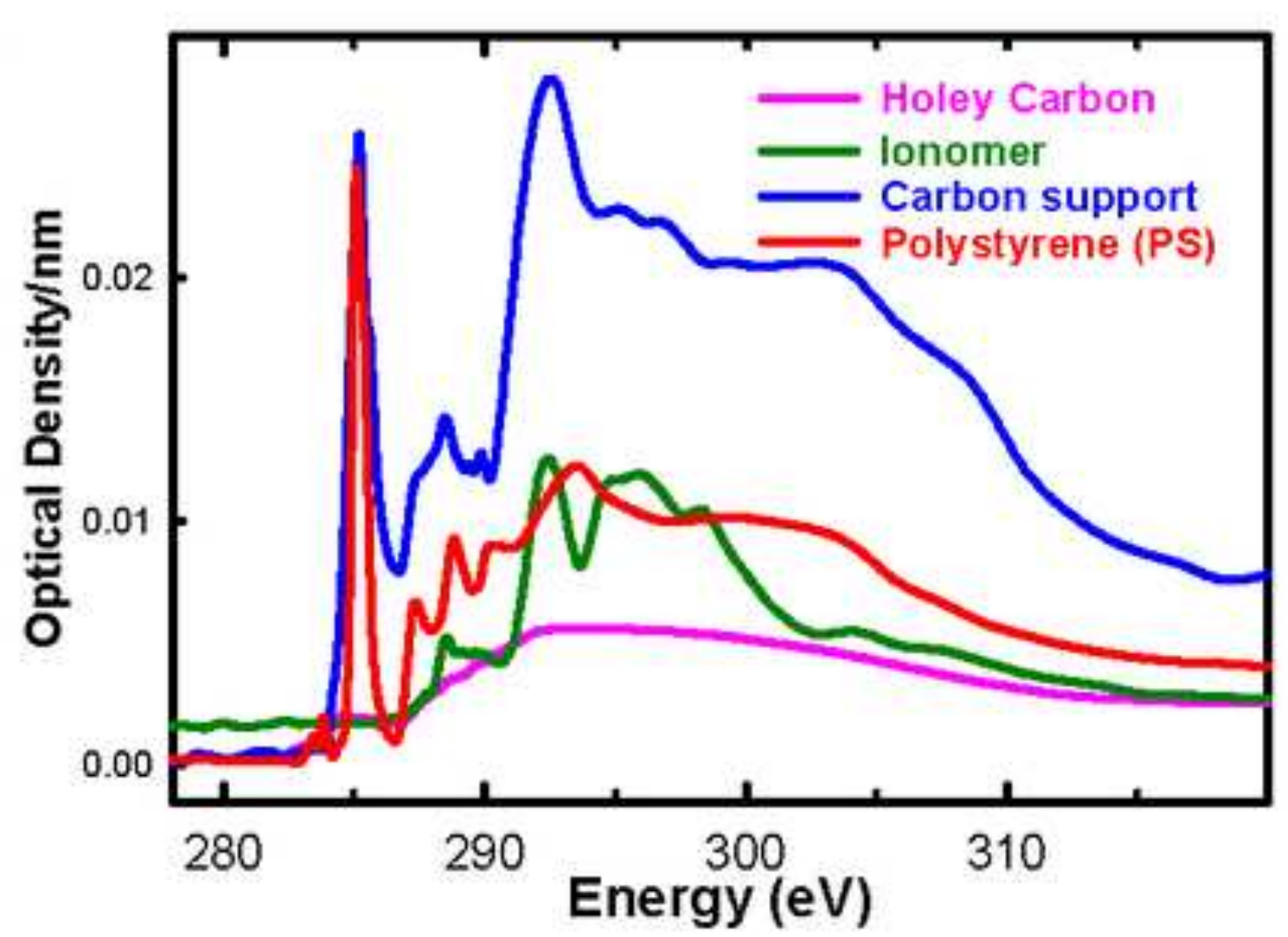

(b) membrane

PS PFSA $\downarrow$ ionomer

C-support holey $C$
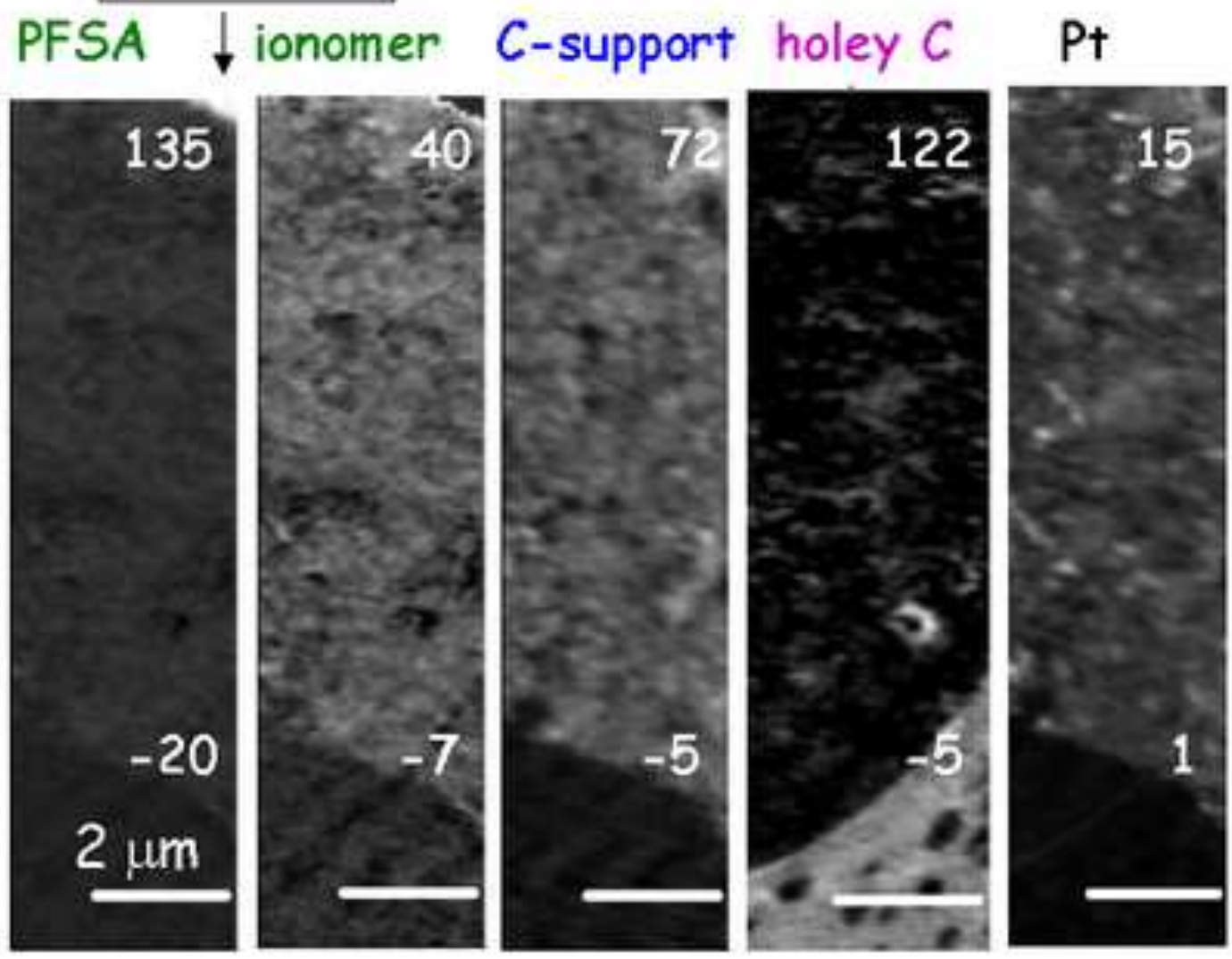


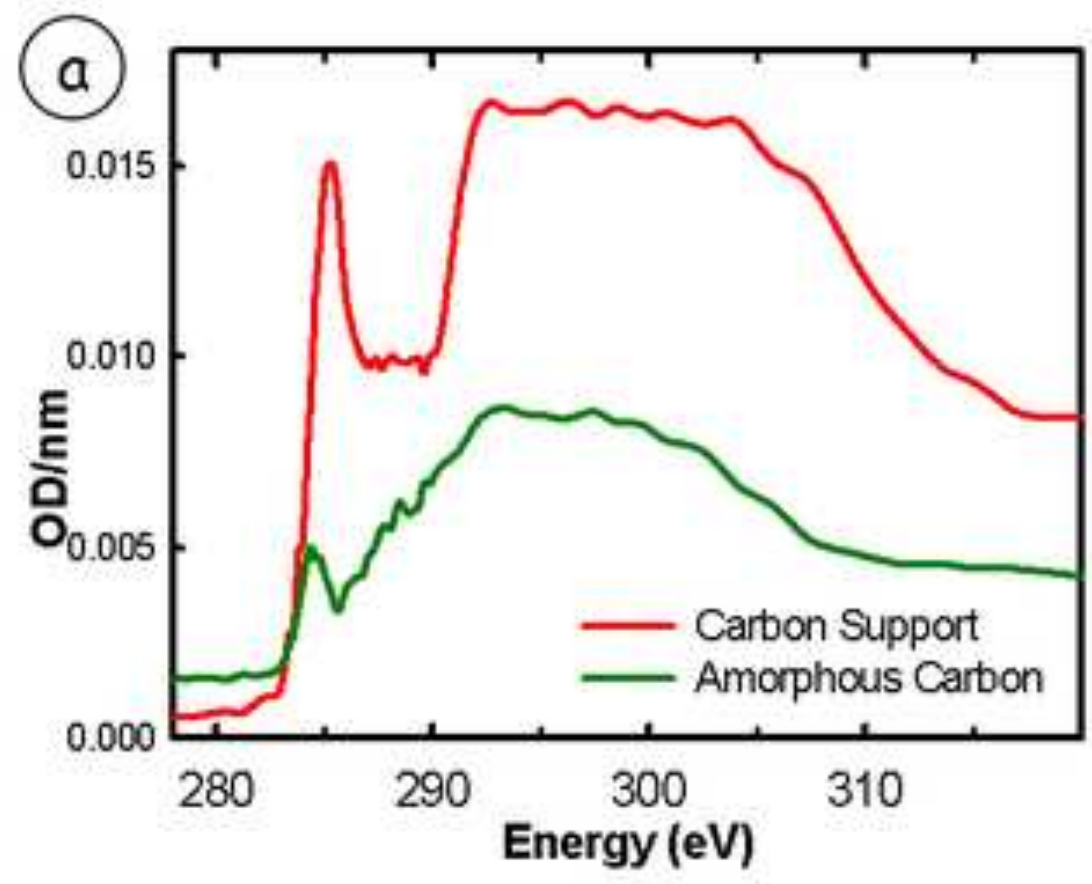

(b) C-support

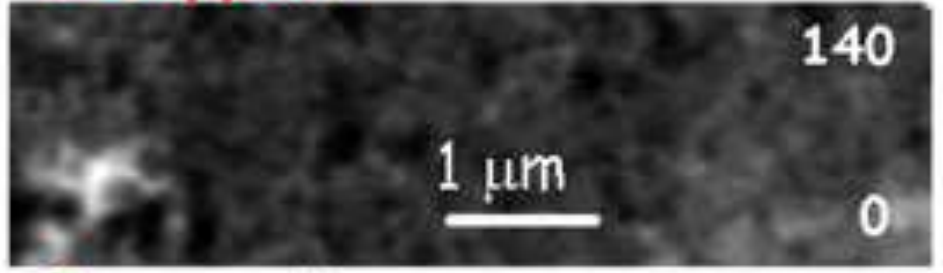

(c) C-amorphous

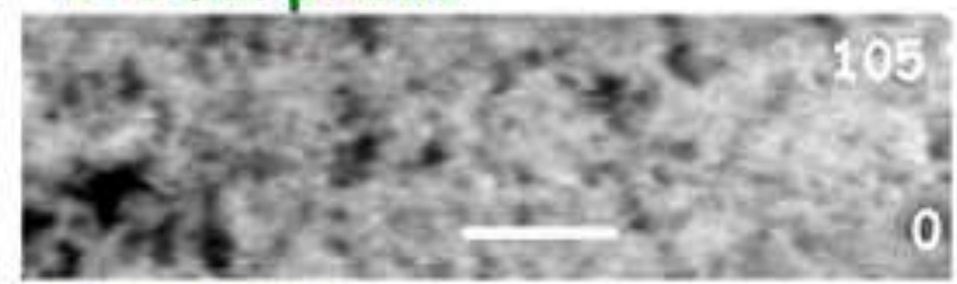

(d) Constant

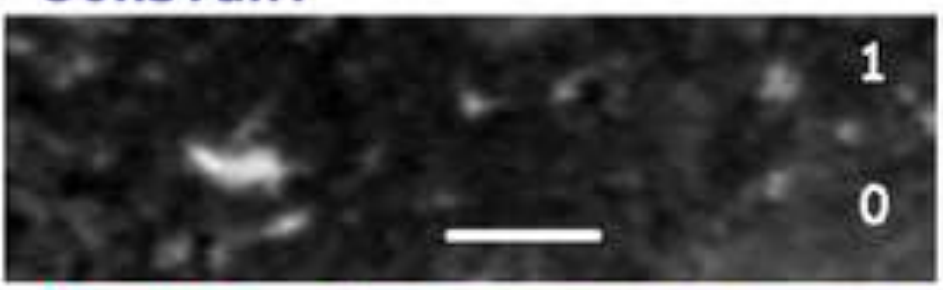

(e) C_supp. C-amorph constant 


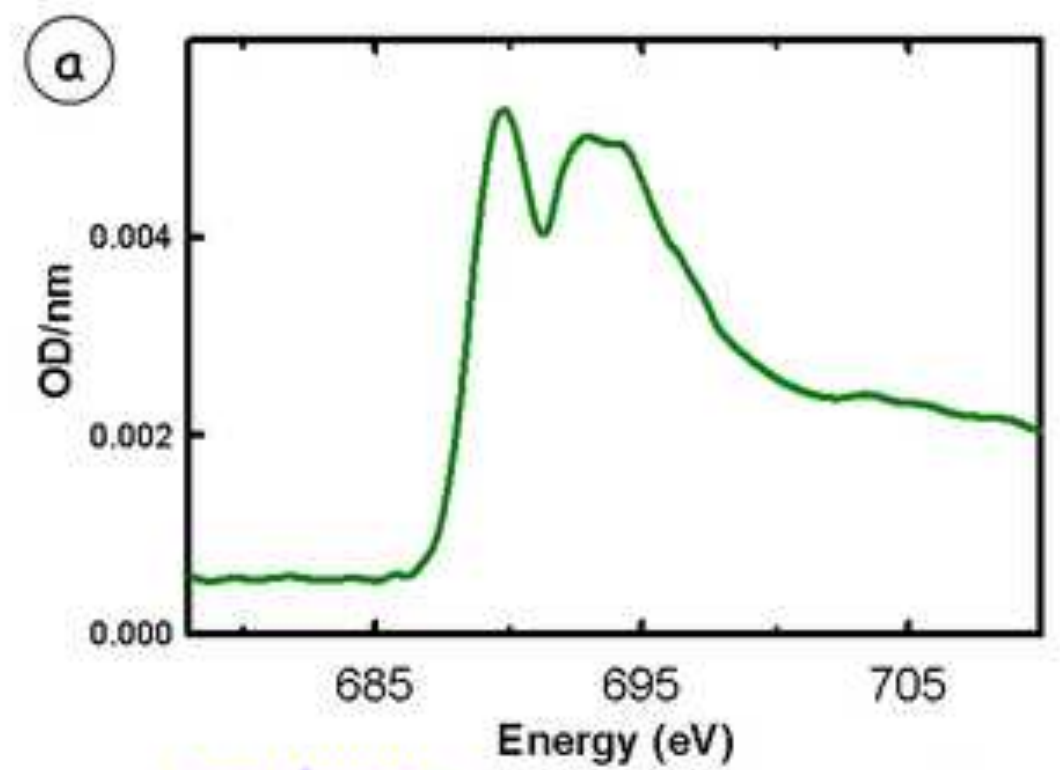

(b) membrane $\mathrm{nm}$ $2 \mu \mathrm{m}$ 184

(c) ionomer

(d) Constant (C-sup, Pt, PS, holey)

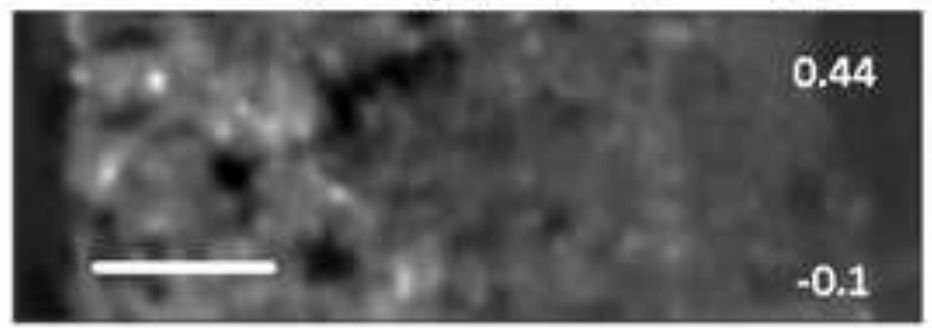

(e) Constant, ionomer, membrane

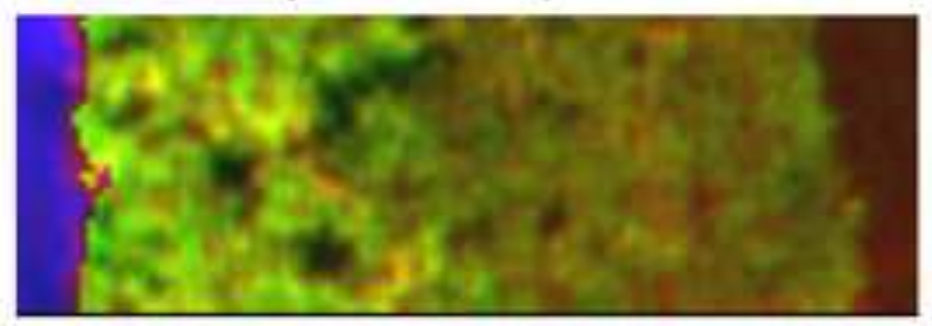




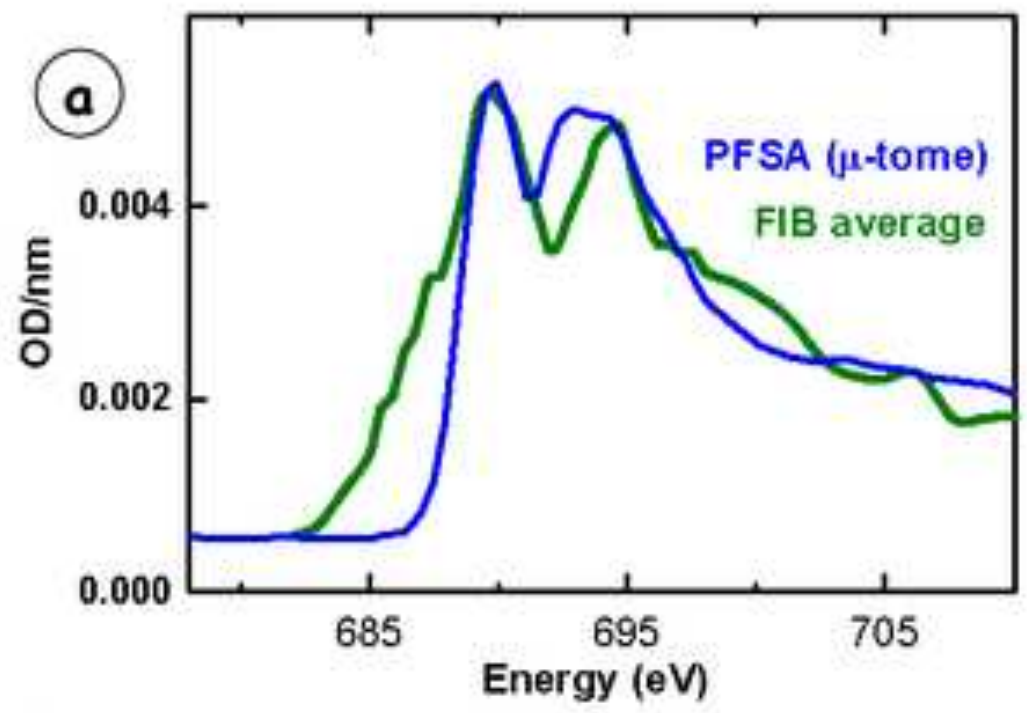

(b) Damaged ionomer

(c) Ionomer

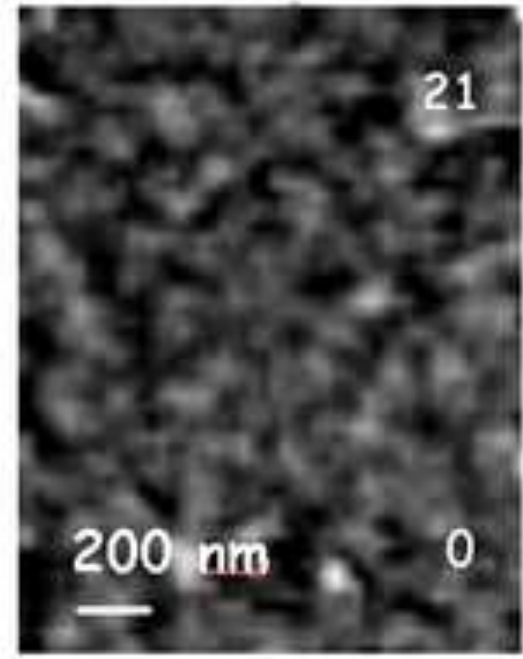

(d) Constant

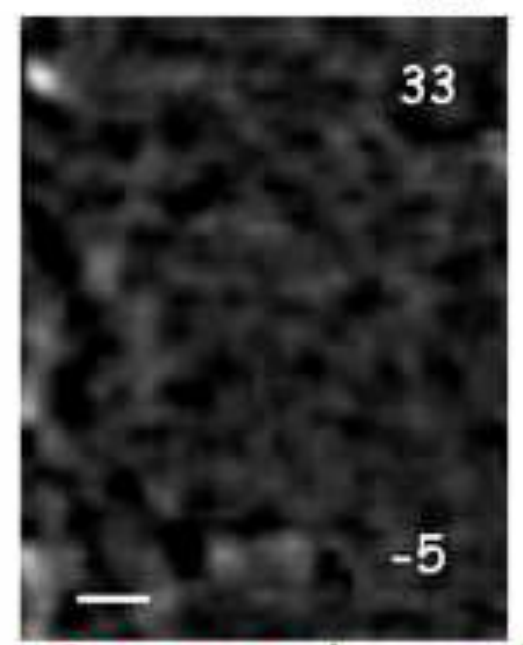

Constant, damaged

(e) ionomer, ionomer
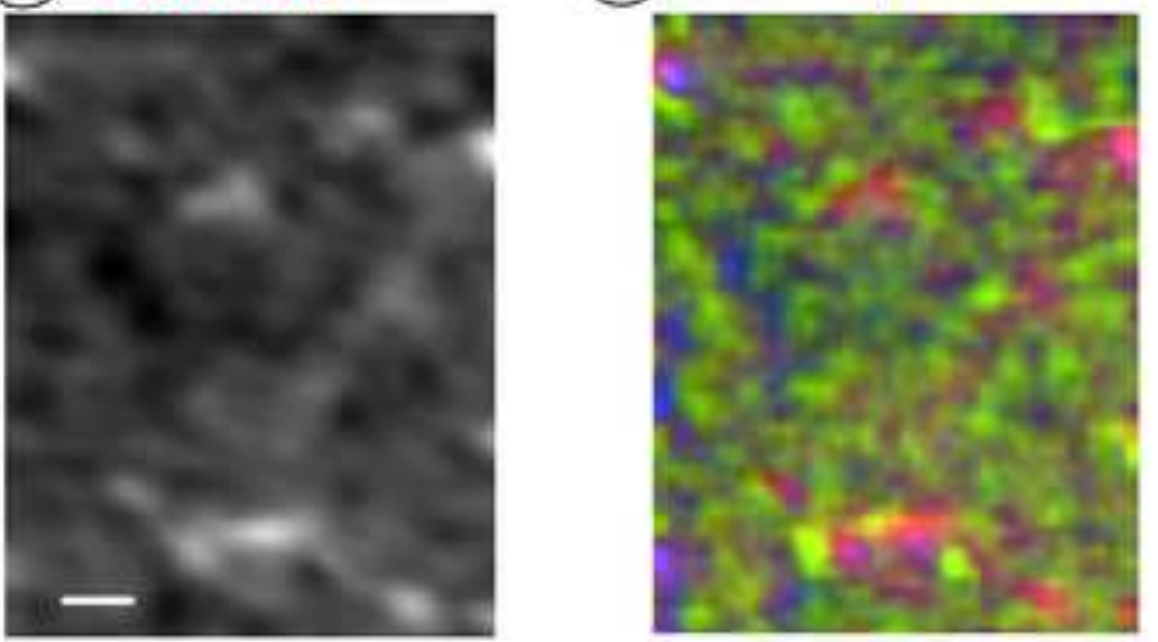
(a) FIB

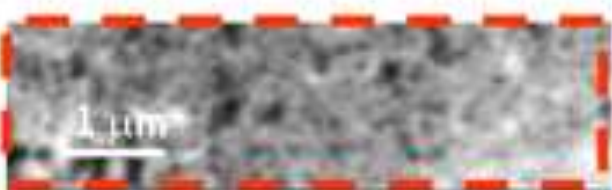

(b) $2 \mu \mathrm{m}$

microtomed
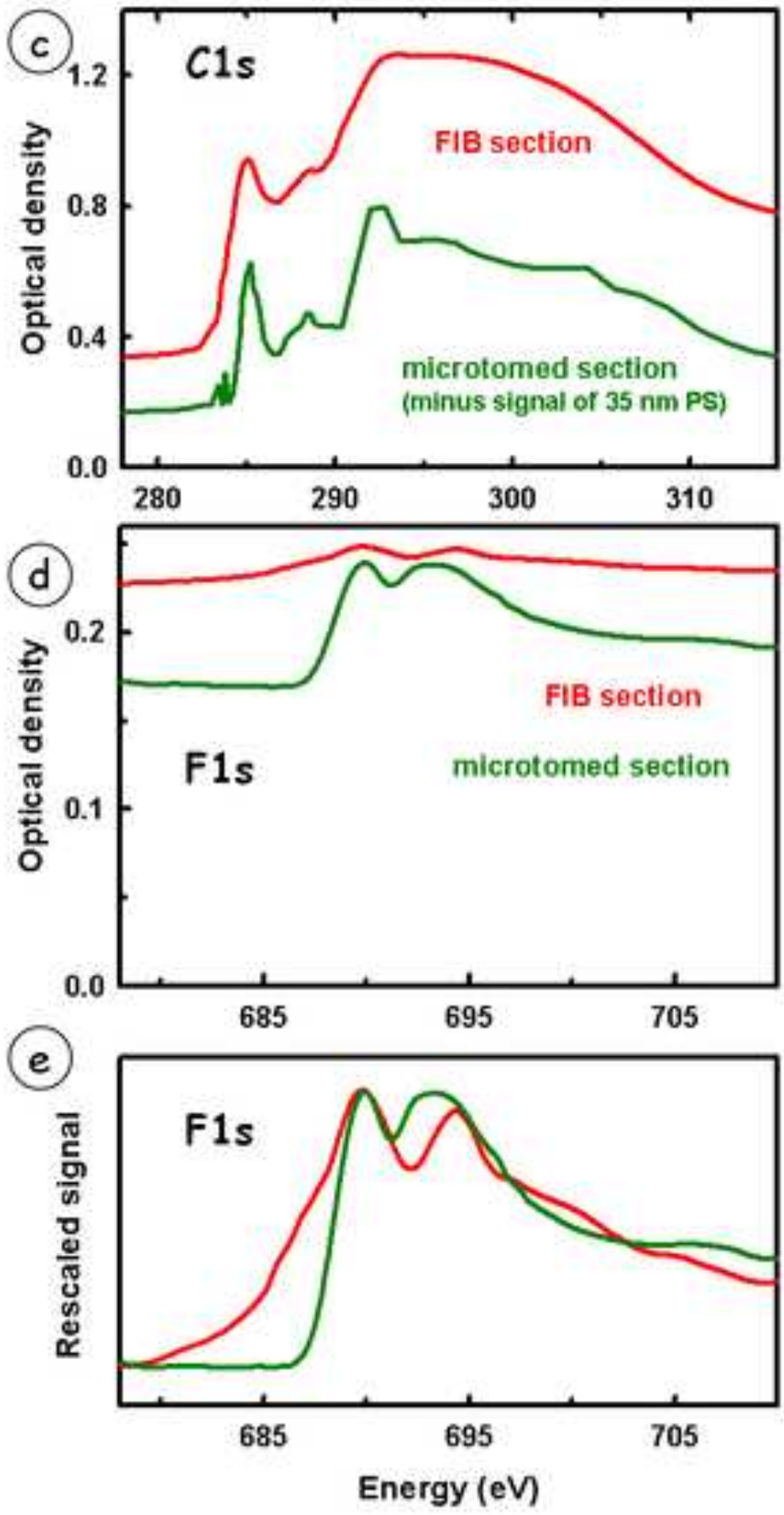


\section{Figure Captions}

Figure 1 Transmission electron microscopy (TEM) of the cathode catalyst. Bright-field images showing Pt nanoparticles and carbon support in (a) the microtomed, and (b) the focused ion beam (FIB) section. The absence of lattice fringes in (b) indicate that the crystalline nature of the carbon support particles is destroyed in the FIB section. (c) Amorphous layer on a carbon particle generated in the FIB milling. All images are on the same spatial scale.

Figure 2 Characterizing Ga contamination in the FIB section. (a) HAADF image and (b) EDX maps (Pt, Ga, C, W, O, F) of the same region of the FIB section. The yellow rectangle in Fig 2a is the area of EDX maps. (c) Reconstructed volume using SIRT with 25 iterations and (d) Orthoslice number 68 (of 141 slices) of the FIB-prepared CCM section showing that Pt particles sit on the surface of the carbon support.

Figure 3 EELS spectrum imaging of the FIB section. (a) Bright field image. The green rectangle indicates the region analysed by EELS mapping. (b) Elemental maps ( $\mathrm{Pt}$ is derived from the pre-C1s signal) and RGB composite map of the Pt (red), F (green) and $\mathrm{C}$ (blue) signals. (c) $\mathrm{C} 1 \mathrm{~s}, \mathrm{O}$ 1s and $\mathrm{F}$ 1s EELS spectra averaged from the entire area. The fluorine signal is low but still distinguishable.

Figure 4 STXM C 1s analysis of the microtomed sample. (a) C 1s absorption spectra of the carbon support, PFSA membrane, polystyrene (embedding medium) and holey carbon plotted on an absolute intensity scale (OD per nm thickness). The spectra were derived from a $\mathrm{C}$ 1s image sequence ( 68 images from 278 to $320 \mathrm{eV}$ ), as described in the text. (b) Quantitative maps (on nm thickness grey scales) for these components derived from a stack fit to the image sequence using the reference spectra plotted in (a). The ionomer map in the cathode region was derived from the full PFSA map by threshold masking and subtracting the area of the membrane in the upper right corner.

Figure 5 STXM C 1s analysis of the FIB section. (a) C 1s absorption spectra of a damaged carbon support region and the dominant, amorphous carbon, derived from a $\mathrm{C}$ 
1s image sequence (68 images from 278 to $320 \mathrm{eV}$ ). Component maps of (b) carbon support, (c) amorphous carbon and (d) constant (catalyst), derived from a stack fit of the $\mathrm{C}$ 1s image sequence of the FIB sample using the reference spectra plotted in (a). (e) color coded composite map indicating that the carbon support is an isolated region and most of the section is amorphous carbon.

Figure 6 STXM F 1s analysis of the microtomed section. (a) F 1s absorption spectrum of PFSA from the membrane on an absolute OD/nm scale; (b), (c) and (d) are maps of the PFSA and constant (non-F components, PS, C-support, Pt) derived from a stack fit to the full F 1s image sequence. The PFSA signal has been split into the membrane and ionomer components by threshold masking. (e) color coded composite showing the membrane (blue), the ionomer (green) and the constant (red).

Figure 7 STXM F $1 \mathrm{~s}$ analysis of the FIB section. (a) Averaged F 1s spectrum of the FIB sample, compared to that of PFSA from the membrane of the microtomed sample. In each case, the F 1s signal from the stack was extracted, a signal corresponding to an extrapolation of the underlying $\mathrm{C} 1 \mathrm{~s}$ signal was subtracted, and the intensity scaled to match the elemental response of fluorine with a density of $1 \mathrm{~g} . \mathrm{cm}^{-3}$. These two spectra were used to fit the F 1s image sequence. (b) Component map for damaged ionomer (based on the average F 1s spectrum of the FIB sample). (c) Component map of 'less damaged ionomer' generated by fit to the reference spectrum for PFSA. The spectrum of the pixels with high signal is presented in supplemental Fig. 1. It differs significantly from that of PFSA. (d) component map for the constant (non-fluorine species) (e) color coded composite indicating the non-fluorinated component (red), less-damaged ionomer (blue), and the damaged ionomer (green).

Figure 8 Spectral comparison of FIB and microtomed samples. (a) STXM OD image at $300 \mathrm{eV}$ of the FIB section - the red dashed rectangle is the area over which the averaged spectrum was extracted. (b) STXM OD image at $300 \mathrm{eV}$ of the microtomed section - the green dashed rectangle is the area over which the averaged spectrum was extracted. (c) Average $\mathrm{C}$ 1s spectra of all of the regions indicated in (a) and (b). The FIB spectrum was 
smoothed. The microtomed spectrum has been modified by subtracting the $\mathrm{C} 1 \mathrm{~s}$ spectrum of $35 \mathrm{~nm}$ of PS, the averaged amount of PS determined in the area over which the averaged spectrum was extracted (see Fig. 5b). This is considered the correct way to compare the $\mathrm{C}$ 1s spectrum of the cathode for the two samples since the FIB section does not contain any embedding medium. (d) Average F 1s spectrum of all area of the regions indicated in (a) and (b). (e) Rescaled overplot of the background subtracted F1s spectra of the FIB and microtomed samples. 